Review

\title{
Association between Blood Pressure, Blood Pressure Variability, and Post-Stroke Cognitive Impairment
}

\author{
Kang-Po Lee ${ }^{1,2} \mathbb{D}$, Alice Y. W. Chang ${ }^{3,4}$ and Pi-Shan Sung ${ }^{1,5, * \mathbb{D}}$ \\ 1 Department of Neurology, National Cheng Kung University Hospital, College of Medicine, \\ National Cheng Kung University, Tainan 704, Taiwan; gavin.righ@gmail.com \\ 2 Department of Neurology, E-DA Hospital, Kaohsiung 824, Taiwan \\ 3 Department of Physiology, College of Medicine, National Cheng Kung University, Tainan 704, Taiwan; \\ aywchang@mail.ncku.edu.tw \\ 4 Institute of Basic Medical Sciences, College of Medicine, National Cheng Kung University, Tainan 704, Taiwan \\ 5 Institute of Clinical Medicine, College of Medicine, National Cheng Kung University, Tainan 704, Taiwan \\ * Correspondence: pishansung@gmail.com; Tel.: +886-6-235-3535-3900
}

check for updates

Citation: Lee, K.-P.; Chang, A.Y.W.; Sung, P.-S. Association between Blood Pressure, Blood Pressure Variability, and Post-Stroke Cognitive Impairment. Biomedicines 2021, 9, 773. https://doi.org/10.3390/

biomedicines 9070773

Academic Editor: Aurel Popa-Wagner

Received: 19 May 2021

Accepted: 29 June 2021

Published: 2 July 2021

Publisher's Note: MDPI stays neutral with regard to jurisdictional claims in published maps and institutional affiliations.

Copyright: (c) 2021 by the authors. Licensee MDPI, Basel, Switzerland. This article is an open access article distributed under the terms and conditions of the Creative Commons Attribution (CC BY) license (https:// creativecommons.org/licenses/by/ $4.0 /)$.

\begin{abstract}
After stroke, dynamic changes take place from necrotic-apoptotic continuum, inflammatory response to poststroke neurogenesis, and remodeling of the network. These changes and baseline brain pathology such as small vessel disease (SVD) and amyloid burden may be associated with the occurrence of early or late poststroke cognitive impairment (PSCI) or dementia (PSD), which affect not only stroke victims but also their families and even society. We reviewed the current concepts and understanding of the pathophysiology for PSCI/PSD and identified useful tools for the diagnosis and the prediction of PSCI in serological, CSF, and image characteristics. Then, we untangled their relationships with blood pressure (BP) and blood pressure variability (BPV), important but often overlooked risk factors for PSCI/PSD. Finally, we provided evidence for the modifying effects of BP and BPV on PSCI as well as pharmacological and non-pharmacological interventions and life style modification for PSCI/PSD prevention and treatment.
\end{abstract}

Keywords: post-stroke cognitive impairment; dementia; stroke; pathophysiology; treatments; blood pressure; blood pressure variability

\section{Introduction}

Stroke ranks second worldwide for mortality and morbidity, and ischemic stroke accounts for $67.1-87 \%$ of all strokes [1-3]. Stroke causes a very large economic burden, approximately EUR 20-27 billion annually in the European region [4], with costs in the U.S. as high as USD 4000 per patient per month [5]. Moreover, in addition to the focal deficit-induced disability, there is the later cognitive impairment and behavioral-emotional dysfunction after stroke, which are easily overlooked. Previous studies suggested 9.2-31.4\% of patients may have poststroke dementia (PSD). Poststroke cognitive impairment (PSCI) may be even higher at $35-47 \%$ [6-10] or as high as $80 \%$, as was seen among a series of Chinese patients [11]. Poststroke patients with cognitive impairment have an independent risk of higher mortality [12]. The most vulnerable cognitive domains affected by stroke are working memory and episodic memory, motor dexterity and verbal fluency, followed by recognition memory $[9,13]$. Regarding the behavioral-emotional dysfunction after stroke, one-third of stroke patients suffer from poststroke depression as well as other neuropsychiatric domains, such as apathy, depression, and sleep disorder [14].

Poststroke cognitive function is not constant but dynamic. Stroke survivors may experience a sharp decline in cognitive function and neuropsychiatric symptoms immediately after a stroke. Early PSCI may become evident as early as 2 weeks afterwards [15]. Some patients may recover and return to their baseline condition between 3 and 6 months, but others do not recover [16]. Except for early PSCI, some individuals may again have 
an accelerated rate of decline, causing late PSCI [17-21]. Regarding which domains are more vulnerable, the results are controversial. One study suggested that, in early PSCI, the impairment is specifically related to the lesion site, whereas late PSCI is more of a global dysfunction; on the other hand, other studies suggested that, with the exception of perceptual motor function, all cognitive domains are impaired in early PSCI, especially memory. In late PSCI, executive and language function as well as visuospatial function may improve from 3 months to 1.5 years after the index stroke $[15,22,23]$, while working memory may eventually recover years after the previous stroke [23].

In this review, we focused on the cerebral changes after a stroke and its relationship with the onset of PSCI. We provided the potentially useful markers to predict the occurrence of PSCI. Then, we untangled the relationships between blood pressure/blood pressure variability (BP/BPV) and PSCI and discussed the potential modifications and interventions.

\section{Method and Data Source}

A Medline (PubMed) literature review was performed by using the following search queries:

- (Post-stroke cognitive impairment) AND (Blood pressure)

- (Poststroke cognitive impairment) AND (Blood pressure)

- (Post-stroke cognitive impairment) AND (Blood pressure variability)

- (Poststroke cognitive impairment) AND (Blood pressure variability)

- (Post-stroke dementia) AND (Blood pressure)

- (Poststroke dementia) AND (Blood pressure)

- (Post-stroke dementia) AND (Blood pressure variability)

- (Poststroke dementia) AND (Blood pressure variability)

- (Cognitive impairment after stroke) AND (Blood pressure variability)

- (Dementia) AND (Blood pressure variability)

Two independent authors (K.-P.L. and P.-S.S.) screened titles and abstracts of the publications. Disagreements were solved by the consensus meeting among the three authors. Duplicated entries, retracted publications, studies on other diseases or focusing on special groups, works on animals or in vitro, studies without statistical analysis, nonEnglish written papers, publications as commentaries, letters and editorials, and any other article that did not fit within the scope of this review were excluded. Articles listed in the references were also reviewed in search of more data. We only considered studies published within the last decade.

A total of 898 results were retrieved. Among them, 47 publications were selected according to the aforementioned inclusion and exclusion criteria. The examination of the references from relevant papers detected five other studies fitting the purpose of this review. Therefore, a total of 52 papers were eventually included (Figure 1). 


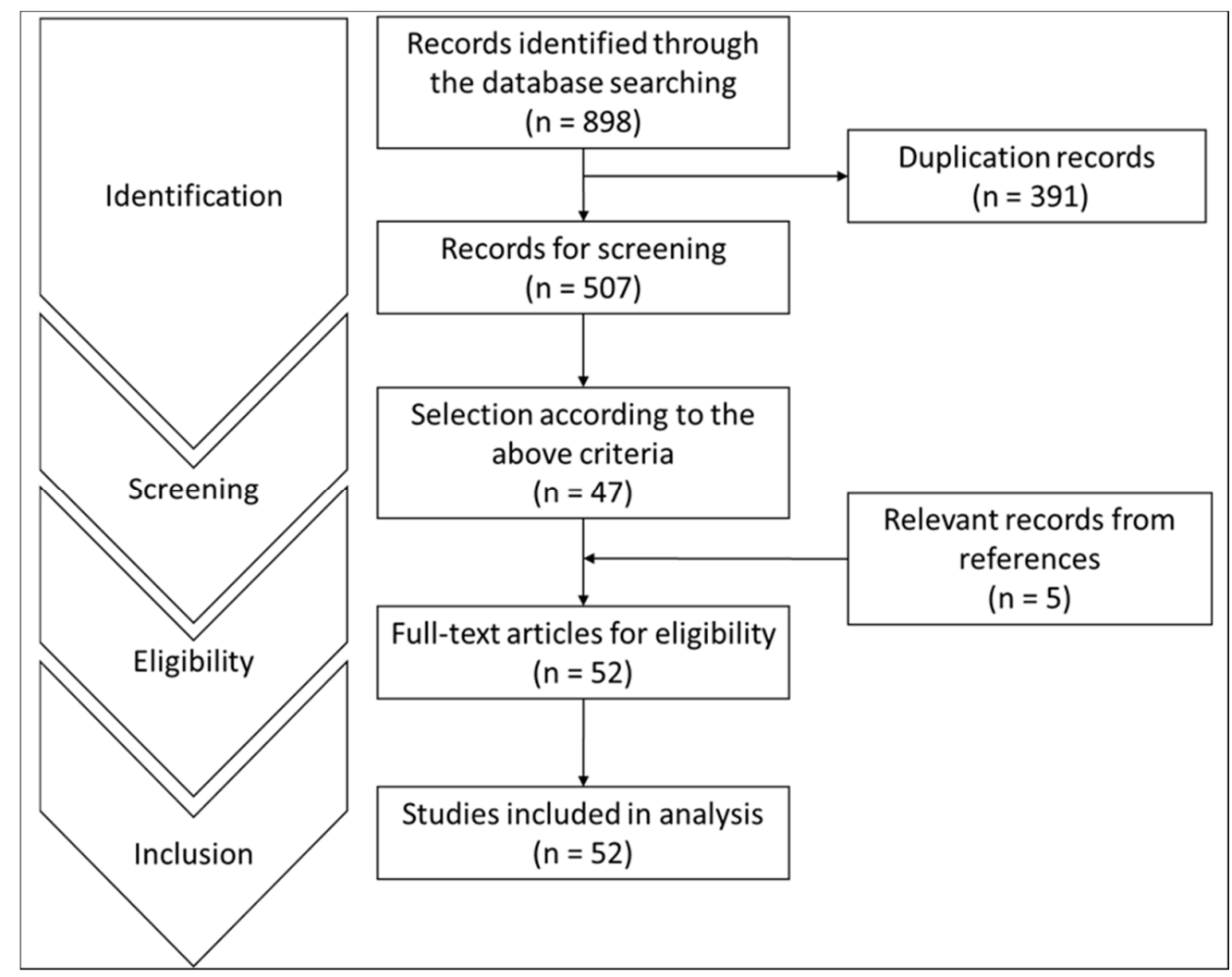

Figure 1. Flow diagram showing the search strategy.

\section{Anatomical, Biochemical and Pathological Changes after Stroke and Their Relationship with PSCI/PSD}

Regarding PSCI/PSD right after stroke, it should first be noted that the location and the size of the infarction matters. A larger infarction size and some specific lesions (e.g., those involving angular gyrus, hippocampus, and frontal cortex) can cause immediate cognitive impairment and even dementia immediately after stroke [24]. A 2009 paper on pathology [25] reported that the neurons became eosinophilic in cytoplasm with a basophilic nuclei right after stroke. The neuron then degraded into debris, and the debris was phagocytized by macrophages, ranging in 1 day to 2 months. Not only neurons but also astrocytes experienced morphological changes. At first, astrocyte swelling was noted, and astrocytes carrying a necrotic aspect became gemistocytic around 2 weeks. Finally, an astrocyte alone with the neurons was necrotized and liquefied. Except for necrotic change, newer studies demonstrated that injured cells underwent apoptosis. They suggested that the so-called apoptosis-necrosis continuum would be a better nomination [26]. Moreover, the vessels also changed within a day, leading to plasma extravasation cellular infiltration, disintegration, and perivascular edema.

The earliest inflammatory response occurred about 4-6 h after onset, starting with leukocyte infiltration and followed by monocytes around $72 \mathrm{~h}$ later. Then, between 1 and 2 weeks, macrophages became prominent. The inflammatory response involved abundant cytokines, both pro-inflammatory (interferon (IFN)- $\gamma$, interleukin (IL)-6, IL-1 $\beta$, tumor necrosis factor (TNF)- $\alpha$ and IL-17) and anti-inflammatory cytokines such as IL-10 [27]. These inflammatory responses may harm the injured brain, causing early cognitive impairment $[28,29]$. A possible explanation is that hippocampal pyramidal cells are selectively more vulnerable than other structures to inflammation. A damaged hippocampus and entorhinal cortex subsequently leads to cognitive impairment [30-32]. The inflammation may not be transient because a neuroimage study demonstrated inflammatory responses beyond 6 months after a stroke [33]. 
Then, the neurogenesis begins. Neurogenesis after stroke occurs in the subventricular zone (SVZ) and the hippocampal subgranular zone (SGZ) [34]. The SVZ is crucial for functional repairment, and the SGZ is crucial for cognitive function [35,36]. The neurogenesis may be both ipsilateral and contralateral to the lesion site. Despite trying to restore their function, aberrant neurogenesis causes further damages. The integration of these new and established cells may remodel the hippocampal network, leading to destabilization and even the loss of pre-stored memory [34,37]. The neurogenesis in SGZ started about 1 week after stroke, peaking around 2 weeks, then gradually recovered to baseline at around 1 month with dendritic maturation $[35,38]$, but the aberrant neurons may have long-lasting effects after stroke [34]. In addition, animal models showed that inhibition of aberrant poststroke hippocampal neurogenesis reduced the remote memory deficits after stroke [38]. Although controversial results exist regarding the issue of poststroke hippocampal neurogenesis and poststroke cognitive outcomes in animal models due to different experimental design and behavior paradigms, poststroke hippocampal damage may be associated with the onset of PSCI/PSD. In humans, accelerated hippocampal atrophy following a stroke was noted both ipsilaterally and contralaterally to the lesion site at 1 year follow up $[39,40]$. Furthermore, more remote events may impair the hippocampal integrity [41].

In addition to the late stroke effect on the hippocampus itself, stroke may disrupt other structures as well. Lesions may involve so-called rich clubs, interrupting the hemispheric connections and the fronto-basal circuits, and may even cause cortical thinning and a decline in total brain volume [42-45]. A functional magnetic resonance imaging (MRI) study in stroke patients showed decreased functional connectivity and regional coherence due to lesions that interrupted white matter circuits, especially in the bilateral mesial temporal lobe and the posterior cingulate gyrus [46-48]. For unknown reasons, white matter integrity on the contralateral side was also affected, which had a more global negative effect on cognition [49].

The development of PSCI/PSD after stroke, therefore, depends on several factors: abovementioned stroke location and stroke volume, related neuronal damage, duration of neuroinflammation, and potentially aberrant hippocampal neurogenesis. In addition, the presence of other pre-existing or subsequent cerebral pathologies is also relevant [50].

The impact of Alzheimer's disease (AD) pathology and changes associated with small vessel disease (SVD) on early and late PSCI is widely discussed. SVD changes and AD pathology are pathological brain changes that may pre-exist or later develop in the brain following a stroke, especially in the elderly. At stroke onset, these pathological changes were possibly subclinical or associated with various severities of prestroke cognitive impairment. In one review, it was suggested that these changes may represent reduced brain resilience to stroke and vascular pathology [17]. However, whether SVD burden, hippocampal atrophy, or amyloid burden correlate the most with PSCI/PSD remains controversial and conflicting [10,51-61]. One review concluded that AD pathology is an important contributor to prestroke dementia. In addition, the presence of AD-like retention shown on amyloid positron emission tomography (PET) was noted in approximately $30 \%$ of patients with early PSCI $[17,62]$, which was significantly higher than that in age-matched nondemented patients following stroke or transient ischemic attack (TIA). Thus, AD pathology may be associated more with early PSCI/PSD. Regarding the impact of SVD, the presence of cerebral microbleeds (CMBs) and white matter hyperintensity (WMHs) were also both shown to be associated with early onset PSCI [17,32,63]. However, regarding late PSCI, evidence may suggest that the presence of severe SVD changes, such as three or more lacunes or severe WMH, was a strong predictor for late PSCI/PSD [64-66]. Another study showed that hippocampal AD pathology did not differ between demented or nondemented poststroke patients and suggested that a non-Alzheimer neurodegenerative process may play a role in dementia following stroke (the mean time from stroke to brain donor was 59-60 months) [55]. AD-like retention of the PET radiotracer in late PSCI was also lower than the retention in patients with early PSCI (19\% over 3 year follow-up vs. approximately $30 \%$ over 3-6 months) [62,67]. However, those with amyloid burden seemed to have a 
more severe and rapid cognitive decline than those without amyloid pathology [67]. The contribution of AD pathology and SVD changes to the appearance of early and late PSCI warrants more investigation.

\section{Biomarkers for PSCI/PSD}

\subsection{Serological Biomarkers}

Unlike animal models, human brain pathologies are precious and scarce. However, due to medical advances, more and more biomarkers are now available not only for vascular dementia but also possibly for predicting the risk of PSCI/PSD. S100B (calcium binding protein $\mathrm{B}$ ), the $\mathrm{S} 100 \mathrm{~B} /$ asymmetric dimethylarginine ratio, and the homocysteine level correlated with baseline small vessel disease burden as leukoaraiosis, lacunar infarct, and deep microbleeds. These findings also lead to a higher risk of vascular dementia (VaD) $[68,69]$. A study demonstrated that, after a strategic stroke, there may be a surge in serum citrulline and dimethylarginine (DMA), and these surges, together with arginine depletion, independently correlate with low MMSE and predict the possibility of cognitive impairment [70]. Higher serum metabolites of glutamine, kynurenine, and lysophosphatidylcholine (18:2), uric acid (UA), low density lipoprotein-cholesterol (LDL-C), vascular endothelial growth factor (VEGF), cortisol, plasma fibrinogen, as well as low plasma amyloid A $\beta 42$, low folic acid, and low vitamin B12 may predict PSCI [18,71-81]. A panel of glutamine, kynurenine, and lysophosphatidylcholines was proposed for predicting PSCI [73]; however, cholesterol level as a predictor is controversial $[80,82]$. Elevated urine formaldehyde, indicating that higher oxidative stress causes PSCI, was reported in one study [83]. Inflammatory markers are also important. Cerebrospinal fluid (CSF) IL-6, -8, -10, and $-1 \beta$ as well as more conventional markers such as C-reactive protein (CRP), highly sensitive CRP (hs-CRP), and rheumatic factors are associated with PSCI [84]. Elevated CRP and hs-CRP during the acute phase may even predict PSCI up to 1 year after stroke $[20,76]$. The APOE $\varepsilon 4$ allele was thought to be an independent risk factor for PSCI and BDNF Val $<$ Met polymorphism as a protector [85-88], but the Bahraini cohort suggested no such correlation between APOE $\varepsilon 4$ and PSCI [89].

\subsection{Imaging Biomarkers}

Almost all stroke patients underwent brain imaging, which told us not only the exact lesion sites but also the baseline brain reserve. Low grey matter, especially over the frontal cortex, WMH burden and location, and brain atrophy strongly predicted future PSCI $[26,60,90]$. In a brain with a high WMH burden, subsequent small infarcts may interrupt vulnerable projections and lead to the thinning of cortical grey matter, thus leading to PSCI $[45,91]$. Regarding PSCI and SVD changes, early PSCI is related to cerebral microbleeds (CMBs) and WMH $[17,32,63]$, and severe WMH or three or more lacunes was associated with the onset of late PSCI [64-66]. A functional MRI using the default mode showed decreased functional connectivity in left medial temporal lobe, posterior cingulate, medial prefrontal cortical areas, and bilateral hippocampus in PSCI patients [47,48,92].

As mentioned above, those with amyloid burden on amyloid PET seemed to have a more severe and rapid cognitive decline after a stroke than those without amyloid pathology [67]. However, another study suggested the occurrence of late PSCI has less connection to amyloid pathology [93]. An ongoing trial, the Determinants of Dementia After Stroke (DEDEMAS) study is still ongoing and may tell us more about the relationship between amyloid burden on amyloid PET and PSCI [94]. Tau PET, on the contrary, may indicate reactive gliosis essential for functional repair. A study suggested that, in amyloid-negative stroke patients, a high uptake of tau was noted both in the lesion and the perilesional area, and the level correlated with the patient's cognitive performance [95].

\section{The Relationship between BP, BPV, and PSCI/PSD}

For a stroke patient, BP control is important, but more and more studies focused on BPV over the last decade. Previous studies showed that higher visit-to-visit BPV predicted 
subsequent stroke in TIA patients [96], higher stroke risk [97], and higher mortality after stroke $[98,99]$. However, the relationship between BP and BPV and cognitive function is less discussed. Here, we pointed out current knowledge on this tangled relationship among BP, BPV, and cognitive impairment.

\subsection{The Association between BP and Cognition}

Regarding BP and dementia risk, population-based studies showed that, from midlife to the geriatric stage, hypertension is correlated with future dementia, including both $\mathrm{AD}$ and $\mathrm{VaD}$ [100-110]. Another large review comprising 17 different systemic reviews concluded that hypertension leads to a higher risk of $\mathrm{VaD}$ and cognitive decline but to less AD [111]. However, other studies suggested that no relation between hypertension and dementia exists [112,113] or that the association is the reverse [114]. Additionally, the relation between cognition and BP may vary by age $[115,116]$.

Higher systolic blood pressure (SBP) may have a negative effect on the hippocampus and the dentate gyrus [117-119], thus negatively influencing the brain reserve. The effect of SBP may also start in early adulthood and predict a lower hippocampal volume in the geriatric stage $[119,120]$. Hypertension might interrupt the connectivity of the temporal lobe, the thalamus, the prefrontal cortex, and especially the hippocampus [117]. Not only hippocampal volume but also hypertension are widely believed to be risk factors for the changes associated with SVD [121-123]. Elevated diastolic BP may be a more specific indicator of the presence of WMHs [124]. In addition to causing SVD changes, in a rat model, hypertension increased amyloid deposition not only in the brain parenchyma but also in the vessel walls in the fronto-parietal region and the hippocampus $[125,126]$. In APOE $\varepsilon 4$ non-demented individuals, elevated blood pressure was also associated with decreased hippocampal volume and a deterioration in cognitive function processing speed [127].

Although there were many observational studies, the true mechanisms underlying the changes are not completely understood. For example, how does hypertension negatively affect our brain despite cerebral autoregulation? It may be that the arterial remodeling in a chronic hypertensive situation causes vessel wall stiffness, which impairs autoregulation. Moreover, in addition to large arteries, hypertension may also damage arterioles and capillaries [128-130]. The above changes may lead to decreased cerebral blood flow (CBF) and cerebral hypoperfusion [131]. In animal models, increased arterial stiffness induced brain dysfunction [130]. A newer concept of the neurovascular unit (NVU) emphasized on the bidirectional talk of neurons and vessels for cerebral autoregulation [132]. A mouse study clearly demonstrated that hypertension may lead to NVU dysregulation and cerebrovascular injury $[133,134]$. Elevated BP may also increase the inflammatory process and breakdown of the blood-brain barrier (BBB) [135-138], which may impair A $\beta$ clearance via the NVU, leading to AD [139].

In addition to the relationship between hypertension and cognitive dysfunction, hypotension would be another potential risk. In patients with down syndrome, lower baseline BP compared with general population was noted [140]. Even with the lack of hypertension or prehypertension, they still have much higher rates of cerebrovascular disease and dementia [141,142]. Another large-scale community-based cohort study with long-term follow-up showed that midlife hypertension and late-life hypotension were associated with 1.62-fold risk of later onset dementia compared with those who were normotensive in mid- to late-life. In addition, declines in BP preceded the onset of mild cognitive impairment or dementia [143]. Whether hypotension plays a role in the etiology of neurodegenerative disease or is a potential concomitant phenomenon of neurodegenerative process warrants future research.

\subsection{The Association between $B P V$ and Cognition}

$\mathrm{BPV}$ is not a constant value but a dynamic value that changes over time, and these changes are classified as ultrashort-term BPV, short-term BPV, and long-term BPV, with each correlating, respectively, to beat-to-beat variations, variation within a day and day-to-day, 
and visit-to-visit variation [144]. The principal indices of these measures include standard deviations, coefficient variations, ranges, night/day ratios, and many others [144,145]. BPV may be another important factor related to cognitive changes, but lack of consensus on BPV methods resulted in heterogeneity among previous epidemiological BPV studies and cognition, and increasing evidence suggests that BPV may be more important than hypertension itself.

In the dementia-free middle-age/old-age population, higher systolic BPV (SBPV) is associated with a higher risk of dementia, and the relationship increases with the duration of higher SBPV [146-148]. In a community-dwelling old population, increased shortterm BPV, SBPV, diastolic BPV (DBPV), and day-to-day BPV but not higher definite BP were associated with cognitive decline and dementia [149-157]. However, controversial results were found in other studies $[158,159]$. As in BP and cognition, age may also play a role. Increased long-term BPV leads to a higher risk of cognitive dysfunction only in the middle-aged population but not in geriatric patients [160]; however, some studies mentioned such correlation in long-term BPV and cognitive impairment in the geriatric population $[153,161,162]$. Both diastolic BPV (DBPV) and SBPV may be associated with more severe deterioration in cognitive function, and there may be a synergistic effect if both are increased [163-166]. In patients with AD, increased short-term BPV and long-term BPV were associated with faster deterioration [150,167]. Higher long-term BPV is associated with AD and amnestic mild cognitive impairment (aMCI) [168]. In patients diagnosed with mild to moderate AD, both higher SBPV and DBPV lead to faster deterioration of cognitive function [169], but in patients with frontotemporal dementia (FTD), no such correlation was noted. A 2018 review suggested that higher long-term BPV is associated with poorer cognitive function in both the normal population and demented patients [170].

However, the true mechanisms underlying the relationships between BPV and the decline in cognitive function remain uncertain [171]. BPV is thought to induce systemic microvascular dysfunction, but the effect on the cerebral vasculature is less certain [172]. In patients with atrial fibrillation, numerous episodes of repetitive hypoperfusion and hyperperfusion were noted in arteries and even in the cerebral capillary system when deep white matter underwent ischemic change [173-175]. The above mechanism may also be considered to apply to high BPV patients as well. Moreover, an animal model showed that increased BPV without hypertension caused vessel wall thickness and subsequent left ventricular hypertrophy [176]. The overall pathophysiology was possibly due to hemodynamic instability induced perfusion imbalance and subsequent inflammatory processes and endothelial damage. These changes were shown to lead to the thickening of blood vessels, arterial stiffness, and deposition of $A \beta[170,177]$. In addition to $A \beta$ deposition, a higher visit-to-visit SBPV may lead to decreased white matter microstructural integrity and an increased rate of brain atrophy [178]. More specifically, long-term BPV may have a remote negative effect on hippocampal volume, especially if it begins in young adulthood [179]. In patients with and without cognitive function complaints, increased daily BPV and day-to-day SBPV were associated with a higher burden of SVD changes, but the correlation was less significant with DBPV [149,180,181]. One study found that increased BPV was associated with total WMH but not with either periventricular or deep WMHs [182]. However, the same group subsequently conducted a meta-analysis showing that BPV was independently associated with a higher SVD burden, especially total WMHs and periventricular WMH but not deep WMH [183]. Another study group demonstrated that a high SBPV was associated with WMH, lacunes, and microbleeds [184]. A metaanalysis of people without dementia showed that BPV, regardless of the time span, was independently correlated with $\mathrm{WMH}$, but the relationship was less evident with lacunes and microbleeds [185]. The association between BPV and SVD changes varied among studies, which was potentially related to different study designs and targeted populations. Table 1 summarized the relationship between BP/BPV and cognitive function. 
Table 1. Summary of the relationship of blood pressure, blood pressure variability, and cognitive function.

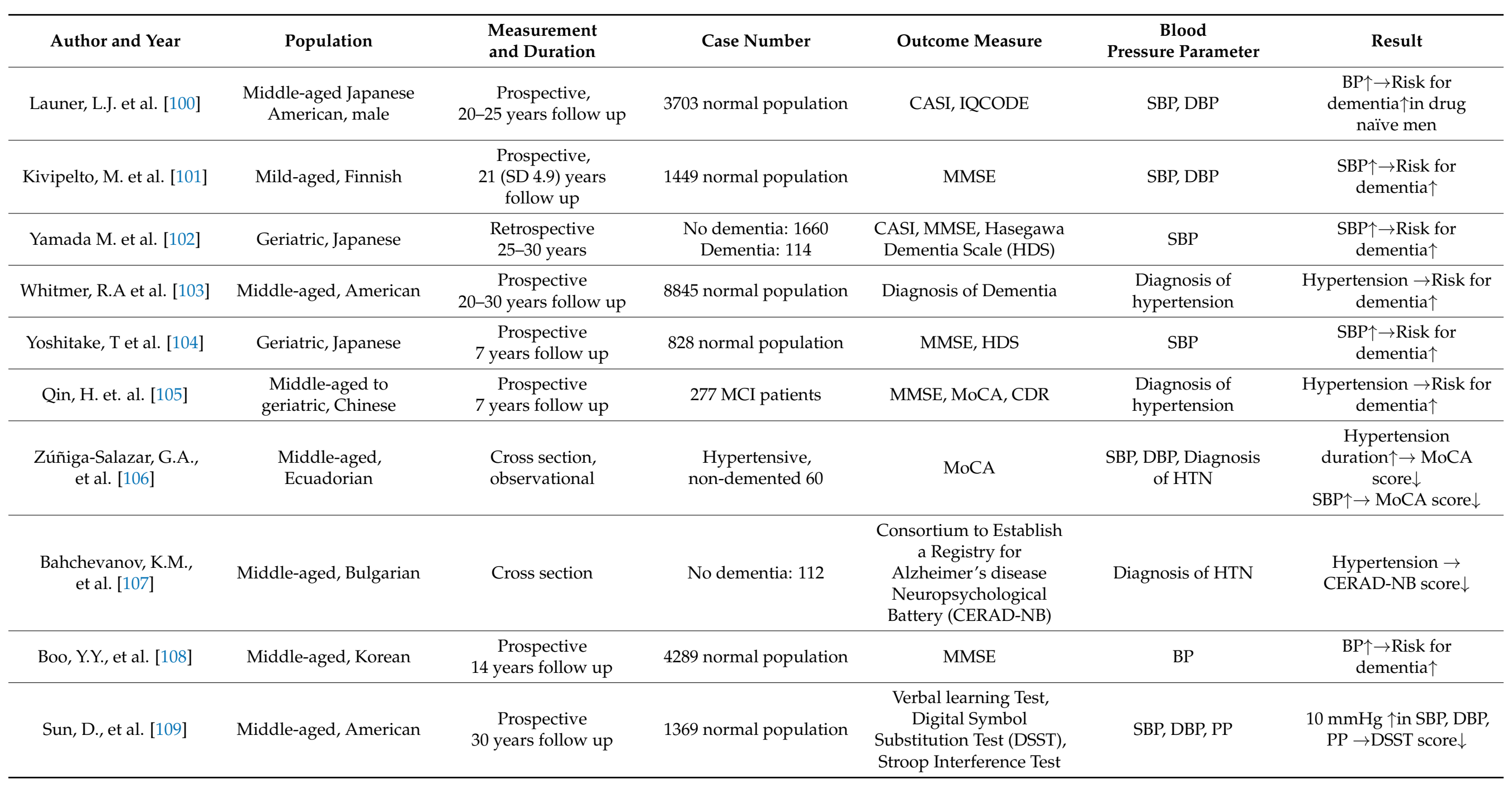


Table 1. Cont.

\begin{tabular}{|c|c|c|c|c|c|c|}
\hline Author and Year & Population & $\begin{array}{l}\text { Measurement } \\
\text { and Duration }\end{array}$ & Case Number & Outcome Measure & $\begin{array}{c}\text { Blood } \\
\text { Pressure Parameter }\end{array}$ & Result \\
\hline $\begin{array}{l}\text { Shim, Y.S. and } \\
\text { H.E. Shin [110] }\end{array}$ & Geriatric, Korean & Cross section & Impaired cognition: 174 & MMSE & SBP, short-term BPV & $\begin{array}{c}\text { SBP } \rightarrow \uparrow \rightarrow \text { Risk for } \\
\text { dementia } \uparrow\end{array}$ \\
\hline Kuller, L.H. et al. [112] & Geriatric, American & Retrospective & 3608 normal population & MMSE, IQCODE & $\begin{array}{l}\text { Diagnosis of } \\
\text { hypertension }\end{array}$ & $\begin{array}{c}\text { No relation between } \\
\text { hypertension } \\
\text { and dementia }\end{array}$ \\
\hline Tyas, S.L. et al. [113] & Geriatric, Canadian & $\begin{array}{c}\text { Prospective } \\
7 \text { years follow up }\end{array}$ & $\begin{array}{l}\text { Normal cognition: } 1335 \\
\text { Impaired cognition: } 42\end{array}$ & Modified MMSE & $\begin{array}{l}\text { Diagnosis of } \\
\text { hypertension }\end{array}$ & $\begin{array}{l}\text { No relation between } \\
\text { hypertension } \\
\text { and dementia }\end{array}$ \\
\hline $\begin{array}{c}\text { Carmona-Abellan, M., } \\
\text { et al. [114] }\end{array}$ & $\begin{array}{c}\text { Middle-aged to geriatric, } \\
\text { Spain }\end{array}$ & $\begin{array}{c}\text { Retrospective } \\
>2.5 \text { years follow up }\end{array}$ & Normal cognition: 2087 & $\begin{array}{c}\text { Diagnosis of dementia, } \\
\text { MCI }\end{array}$ & SBP, DBP & $\begin{array}{c}\downarrow \mathrm{SBP}, \mathrm{BP} \rightarrow \text { Risk of } \\
\text { cognitive impairment } \uparrow\end{array}$ \\
\hline Hestad, K., et al. [115] & $\begin{array}{l}\text { Middle-aged to geriatric, } \\
\text { Norwegian }\end{array}$ & $\begin{array}{c}\text { Prospective } \\
8 \text { years follow up }\end{array}$ & 4465 normal population & $\begin{array}{l}\text { MMSE, Digit symbol } \\
\text { Test, Twelve-word test }\end{array}$ & $\mathrm{BP}$ & $\begin{array}{c}\text { Male } \leq 65 \uparrow \mathrm{SBP}, \uparrow \mathrm{DBP} \rightarrow \\
\text { Cognition } \downarrow \text {; reverse in } \\
\text { male }>65 \\
\text { Female } \leq 65 \uparrow \mathrm{SBP} \rightarrow \\
\text { Cognition } \uparrow ; \text { reverse in } \\
\text { female }>65\end{array}$ \\
\hline Feng, R. et al. [117] & UK biobank & Cross section & $\begin{array}{c}\text { Hypertensive: } 2720 \\
\text { Normal BP: } 12366\end{array}$ & $\begin{array}{l}\text { prospective memory, } \\
\text { numeric memory, fluid } \\
\text { intelligence, } \\
\text { reaction time }\end{array}$ & $\begin{array}{l}\text { Diagnosis of } \\
\text { hypertension }\end{array}$ & $\begin{array}{c}\text { Hypertension } \rightarrow \text { Risk for } \\
\text { dementia } \uparrow\end{array}$ \\
\hline Li, H. et al. [118] & Middle-aged, Chinese & Cross section & cognitive impairment: 59 & $\begin{array}{l}\text { MoCA, Stroop test, } \\
\text { Verbal fluency test }\end{array}$ & $\begin{array}{c}\text { SBP, SBP } \\
\text { variability (SBPV) }\end{array}$ & $\begin{array}{l}\uparrow \mathrm{SBP}, \mathrm{SBP} \text { variability } \rightarrow \\
\downarrow \text { dentate gyrus volume }\end{array}$ \\
\hline Walker, K.A., et al. [143] & Middle-aged, American & $\begin{array}{c}\text { Prospective } \\
24 \text { years follow up }\end{array}$ & 4761 normal population & $\begin{array}{c}\text { Comprehensive } \\
\text { neuropsychological } \\
\text { battery, CDR, diagnosis } \\
\text { of dementia }\end{array}$ & SBP, DBP & $\begin{array}{l}\text { midlife hypertension } \\
\text { and late-life hypotension } \\
\rightarrow \text { risk for dementia } \uparrow\end{array}$ \\
\hline Ma, Y. et al. [146] & Geriatric, Dutch & $\begin{array}{l}\text { Prospective } \\
14 \text { years follow up }\end{array}$ & Normal cognition: 5273 & MMSE & $\begin{array}{l}\text { Long-term SBPV, } \\
\text { Long-term DBPV }\end{array}$ & $\begin{array}{c}\uparrow \text { long-term } \\
\mathrm{SBPV}, \uparrow \mathrm{DBPV} \rightarrow \text { Risk of } \\
\text { cognitive impairment } \uparrow\end{array}$ \\
\hline
\end{tabular}


Table 1. Cont.

\begin{tabular}{|c|c|c|c|c|c|c|}
\hline Author and Year & Population & $\begin{array}{l}\text { Measurement } \\
\text { and Duration }\end{array}$ & Case Number & Outcome Measure & $\begin{array}{c}\text { Blood } \\
\text { Pressure Parameter }\end{array}$ & Result \\
\hline Yano, Y. et al. [148] & Middle-aged, American & $\begin{array}{c}\text { Prospective } \\
25 \text { years follow up }\end{array}$ & 15792 normal population & $\begin{array}{c}\text { Delay Word Recall Test, } \\
\text { Digit Symbol } \\
\text { Substitution Test, Word } \\
\text { Fluency Test }\end{array}$ & SBP, DBP, SBPV, DBPV, & $\begin{array}{l}\uparrow S B P V, \uparrow D B P V \rightarrow \\
\text { Cognitive function } \downarrow \\
\text { SBP, DBP } \rightarrow \text { No } \\
\text { association }\end{array}$ \\
\hline Godai Si, K. et al. [149] & Geriatric, Japanese & Cross section & 111 normal population & MoCA & Short-term BPV & $\begin{array}{c}\uparrow \text { short-term BPV } \rightarrow \text { Risk } \\
\text { of cognitive } \\
\text { impairment } \uparrow\end{array}$ \\
\hline $\begin{array}{l}\text { de Haus, R.A.A. } \\
\text { et al. [150] }\end{array}$ & Geriatric, Dutch & $\begin{array}{c}\text { Prospective } \\
1.5 \text { years follow up }\end{array}$ & $\begin{array}{l}460 \text { mild-to-moderate } \\
\text { AD patients }\end{array}$ & $\begin{array}{c}\text { Alzheimer's Disease } \\
\text { Assessment } \\
\text { Scale-cognitive subscale } \\
\text { (ADAS-cog) }\end{array}$ & $\begin{array}{l}\text { Long-term BPV } \\
\text { Short-term BPV }\end{array}$ & $\begin{array}{c}\uparrow \text { short-term BPV, } \\
\uparrow \text { long-term BPV } \rightarrow \text { Risk } \\
\text { of cognitive } \\
\text { impairment } \uparrow\end{array}$ \\
\hline Oishi, E. et al. [151] & Geriatric, Japanese & $\begin{array}{c}\text { Prospective } \\
5 \text { years follow up }\end{array}$ & 1674 normal population & MMSE, HDS & $\begin{array}{c}\text { Day-to-day BPV } \\
\text { Daily BPV } \\
\text { SBP }\end{array}$ & $\begin{array}{c}\uparrow \text { Day-to-day BPV } \rightarrow \text { risk } \\
\text { of cognitive } \\
\text { impairment } \uparrow \\
\uparrow \mathrm{SBP} \rightarrow \text { Risk of } \mathrm{VaD} \uparrow\end{array}$ \\
\hline Fujiwara, T. et al. [153] & Geriatric, Japanese & $\begin{array}{c}\text { Prospective } \\
1 \text { year follow up }\end{array}$ & 524 normal population & Working memory test & $\begin{array}{l}\text { Short-term BPV } \\
\text { Long-term BPV }\end{array}$ & $\begin{array}{c}\uparrow \mathrm{BPV} \rightarrow \text { Risk of } \\
\text { cognitive impairment } \uparrow\end{array}$ \\
\hline Liu, Z. et al. [154] & Geriatric, Chinese & $\begin{array}{c}\text { Prospective } \\
2.3 \text { years follow up }\end{array}$ & 248 normal population & MMSE & SBPV & $\begin{array}{c}\uparrow \text { SBPV } \rightarrow \text { Speed of } \\
\text { cognitive impairment } \uparrow\end{array}$ \\
\hline McDonald, C. et al. [155] & Geriatric, UK & $\begin{array}{c}\text { Prospective } \\
5 \text { years follow up }\end{array}$ & 353 normal population & $\begin{array}{c}\text { MMSE, Cambridge } \\
\text { Cognitive Examination } \\
\text { (CAMCOG) }\end{array}$ & Day-time BPV & $\begin{array}{c}\uparrow \mathrm{SBPV}, \mathrm{DBPV} \rightarrow \text { Risk of } \\
\text { cognitive impairment } \uparrow, \\
\text { Speed of cognitive } \\
\text { impairment } \uparrow\end{array}$ \\
\hline Nagai, M. et al. [156] & Geriatric, Japanese & $\begin{array}{c}\text { Prospective } \\
1 \text { year follow up }\end{array}$ & $\begin{array}{l}201 \text { patients high risk for } \\
\text { CVD }\end{array}$ & MMSE & $\begin{array}{l}\text { Long-term BPV } \\
\text { Short-term BPV }\end{array}$ & $\begin{array}{c}\uparrow \text { long-term BPV } \rightarrow \text { Risk } \\
\text { of cognitive } \\
\text { dysfunction } \uparrow\end{array}$ \\
\hline
\end{tabular}


Table 1. Cont.

\begin{tabular}{|c|c|c|c|c|c|c|}
\hline Author and Year & Population & $\begin{array}{l}\text { Measurement } \\
\text { and Duration }\end{array}$ & Case Number & Outcome Measure & $\begin{array}{c}\text { Blood } \\
\text { Pressure Parameter }\end{array}$ & Result \\
\hline Yildirim, E. et al. [157] & Geriatric, Turkish & $\begin{array}{c}\text { Prospective } \\
1 \text { year follow up }\end{array}$ & $\begin{array}{l}435 \text { hypertensive } \\
\text { patients }\end{array}$ & $\begin{array}{l}\text { Standardized mini } \\
\text { mental test (sMMT) }\end{array}$ & Short-term BPV & $\begin{array}{c}\uparrow \text { short-term BPV } \rightarrow \text { Risk } \\
\text { of cognitive } \\
\text { dysfunction } \uparrow\end{array}$ \\
\hline Tsang, S. et al. [158] & $\begin{array}{c}\text { Middle-aged to geriatric, } \\
\text { American }\end{array}$ & Cross section & Normal cognition: 94 & $\begin{array}{c}\text { MMSE, Computer } \\
\text { Assessment of Mild } \\
\text { Cognitive Impairment } \\
\text { (CAMCI) }\end{array}$ & SBPV, DBPV & $\begin{array}{l}\text { No association of BPV } \\
\text { and dementia }\end{array}$ \\
\hline $\begin{array}{l}\text { van Middelaar, T. } \\
\text { et al. [159] }\end{array}$ & Geriatric, Dutch & $\begin{array}{l}\text { Prospective } \\
6.4 \text { (SD 0.8) years } \\
\text { follow up }\end{array}$ & 2305 normal population & MMSE & Long-term BPV & $\begin{array}{l}\text { No association of BPV } \\
\text { and dementia }\end{array}$ \\
\hline Qin, B. et al. [160] & $\begin{array}{l}\text { Middle-aged to } \\
\text { geriatric, Chinese }\end{array}$ & $\begin{array}{c}\text { Prospective } \\
3.2 \text { years follow up }\end{array}$ & 976 normal population & $\begin{array}{l}\text { Telephone Interview for } \\
\text { Cognitive } \\
\text { Status-modified } \\
\text { (TICS-m) }\end{array}$ & Long-term BPV & $\begin{array}{c}\uparrow \text { long-term BPV } \rightarrow \text { Risk } \\
\text { of cognitive } \\
\text { dysfunction } \uparrow \text { in } \\
\text { middle-aged; but no } \\
\text { association in } \\
\text { geriatric patients }\end{array}$ \\
\hline $\begin{array}{l}\text { Alpérovitch, A. } \\
\text { et al. [161] }\end{array}$ & Geriatric, French & $\begin{array}{c}\text { Prospective } \\
8 \text { years follow up }\end{array}$ & 6506 normal population & MMSE & Long-term SBPV & $\begin{array}{c}\uparrow \mathrm{SBPV} \rightarrow \text { Risk of } \\
\text { dementia } \uparrow\end{array}$ \\
\hline Epstein, N.U. et al. [162] & $\begin{array}{c}\text { Middle-aged to geriatric, } \\
\text { American }\end{array}$ & $\begin{array}{c}\text { Prospective } \\
3 \text { years follow up }\end{array}$ & $\begin{array}{l}\text { Normal cognition: } 181 \\
\text { MCI } 247\end{array}$ & $\begin{array}{c}\text { MMSE, CDR, } \\
\text { ADAS-COG, Trail B, } \\
\text { Digit Symbol Test, Rey } \\
\text { auditory learning test }\end{array}$ & Long-term BPV & $\begin{array}{c}\uparrow \text { Long-term SBPV } \rightarrow \\
\text { Risk cognitive } \\
\text { dysfunction } \uparrow\end{array}$ \\
\hline Zhou, T.L. et al. [163] & $\begin{array}{c}\text { Middle-aged to geriatric, } \\
\text { American }\end{array}$ & Cross section & 1804 normal population & $\begin{array}{l}\text { Memory function } \\
\text { Processing speed } \\
\text { Executive function }\end{array}$ & $\begin{array}{l}\text { Ultra-short BPV } \\
\text { Daily BPV } \\
\text { Long-term BPV }\end{array}$ & $\begin{array}{c}\uparrow \text { ultra-short, } \uparrow \text { daily } \\
\text { SBPV, } \uparrow \text { DBPV } \rightarrow \\
\text { Cognitive performance } \downarrow\end{array}$ \\
\hline Rouch, L. et al. [164] & Geriatric, French & $\begin{array}{c}\text { Prospective } \\
3 \text { years follow up }\end{array}$ & 3319 normal population & MMSE & $\begin{array}{c}\text { Long-term SBPV } \\
\text { Long-term DBPV } \\
\text { MAP, PPV }\end{array}$ & $\begin{array}{c}\uparrow \text { long-term SBPV, } \\
\text { long-term DBPV } \rightarrow \text { Risk } \\
\text { of dementia } \uparrow\end{array}$ \\
\hline
\end{tabular}


Table 1. Cont.

\begin{tabular}{|c|c|c|c|c|c|c|}
\hline Author and Year & Population & $\begin{array}{l}\text { Measurement } \\
\text { and Duration }\end{array}$ & Case Number & Outcome Measure & $\begin{array}{c}\text { Blood } \\
\text { Pressure Parameter }\end{array}$ & Result \\
\hline Sabayan, B. et al. [165] & Geriatric, European & $\begin{array}{c}\text { Prospective } \\
3.2 \text { years follow up }\end{array}$ & $\begin{array}{c}5461 \text { patients with CV } \\
\text { risk without cognitive } \\
\text { impairment }\end{array}$ & $\begin{array}{l}\text { Stroop color and word } \\
\text { test, letter-digit coding } \\
\text { test, picture-word } \\
\text { learning test }\end{array}$ & $\begin{array}{c}\text { SBP, DBP } \\
\text { Long-term SBPV } \\
\text { Long-term DBPV } \\
\text { DBPV }\end{array}$ & $\begin{array}{c}\uparrow \text { long-term BPV } \rightarrow \text { Risk } \\
\text { of cognitive } \\
\text { impairment } \uparrow\end{array}$ \\
\hline Yoo, J.E. et al. [166] & Korean data base & $\begin{array}{c}\text { Retrospective } \\
6.2 \text { years follow up }\end{array}$ & $7,844,814$ patients & Diagnosis of dementia & SBPV, DBPV & $\begin{array}{c}\uparrow \text { long-term } \mathrm{BPV} \rightarrow \text { Risk } \\
\text { of all dementia } \uparrow, \mathrm{AD} \uparrow, \\
\operatorname{VaD} \uparrow\end{array}$ \\
\hline Lattanzi, S. et al. [167] & Geriatric, Italian & $\begin{array}{c}\text { Prospective } \\
12 \text { months follow up }\end{array}$ & $\begin{array}{l}240 \text { patients with } \\
\text { dementia }\end{array}$ & MMSE & SBP, DBP, SBPV, DBPV & $\begin{array}{c}\uparrow \mathrm{SBPV} \rightarrow \text { Progression of } \\
\text { cognitive decline } \uparrow\end{array}$ \\
\hline Sible, I.J. et al. [168] & $\begin{array}{l}\text { Middle-aged to geriatric } \\
\text { American and Canadian }\end{array}$ & $\begin{array}{c}\text { Prospective } \\
12 \text { months follow up }\end{array}$ & $\begin{array}{c}681 \text { normal cognition } \\
479 \mathrm{MCI} \\
261 \mathrm{AD}\end{array}$ & MMSE, CDR & $\begin{array}{l}\text { SBP, DBP, BPV, } \\
\text { Long-term BPV }\end{array}$ & $\begin{array}{c}\text { In AD patients there } \\
\text { is } \uparrow \mathrm{BPV} \\
\uparrow \mathrm{BPV} \rightarrow \uparrow \text { progression } \\
\text { in MCI }\end{array}$ \\
\hline Lattanzi, S. et al. [169] & Geriatric, Italian & $\begin{array}{c}\text { Prospective } \\
12 \text { months follow up }\end{array}$ & $\begin{array}{l}248 \text { AD } \\
81 \text { FTD }\end{array}$ & MMSE & $\mathrm{BP}, \mathrm{BPV}$ & $\begin{array}{c}\uparrow \mathrm{SBPV} \rightarrow \uparrow \text { progression } \\
\text { in } \mathrm{AD}\end{array}$ \\
\hline
\end{tabular}

$\uparrow$, increase; $\downarrow$. decrease; $\rightarrow$ leads to. 


\subsection{Influence of $B P / B P V$ in PSCI and PSD}

Regarding BP/BPV and PSCI, the situation is more complicated but less frequently discussed. A higher SBP upon stroke admission seemed to be correlated with a higher risk of PSCI [186]. In the acute phase within 7 days, there seemed to be a U-shaped association regarding SBP and poststroke cognition [187]. In the subacute phase of less than 90 days, elevated SBP was thought to be a risk factor for PSCI with or without DBP differences, and DBP was significantly inversely related to cognition after adjusting for age, education, and races [188]. In the chronic phase, home BP is more important than clinic BP, and higher morning $\mathrm{BP}$ and bedtime BP were specifically related to a higher risk for recurrent ischemic events and PSCI [189]. However, the Sydney study suggests that hypertension is not a risk factor for PSCI between 3 and 6 months [190]. A study on BP control also showed no beneficial effect on cognition for different levels of SBP [191], and another demonstrated that the correlation between hypertension and PSCI was established only when patients had concurrent hyperhomocysteinemia [192]. In fact, a meta-analysis stated that $\mathrm{BP}$ and poststroke cognitive performance had no correlation [7]. However, increased BPV during the early phase of stroke may predict PSCI $[193,194]$. The subgroup analysis of the "PreventIon of CArdiovascular events in iSchemic Stroke patients with high risk of cerebral hemOrrhage (PICASSO)" trial showed that BPV, not SBP, was associated with faster cognitive decline after a stroke [195].

As mentioned above, BP or BPV may have a potential negative effect on poststroke cognitive function. BP and BPV are also potentially related to the major pathophysiology of PSCI, including AD pathology, SVD changes, and ischemic neurodegeneration. Further study may be warranted to investigate the impact of $\mathrm{BP} / \mathrm{BPV}$ on the progression of PSCI/PSD. Table 2 summarized the relationship of BP/BPV and cognitive function after stroke. 
Table 2. Summary of the relationship among blood pressure, blood pressure variability, poststroke cognitive impairment, and poststroke dementia.

\begin{tabular}{|c|c|c|c|c|c|c|}
\hline Author and Year & Follow up after Stroke & Case Numbers & Special Condition & Outcome Measure & Blood Pressure & Result \\
\hline Gong, L. et al. [186] & $\begin{array}{l}\text { Prospective, } 6 \text { months } \\
\text { follow up }\end{array}$ & 141 & Early PSCI & MoCA & SBP & $\begin{array}{l}\uparrow \text { Acute phase SBP } \rightarrow \downarrow \\
\text { Cognitive performance }\end{array}$ \\
\hline He, M. et al. [187] & $\begin{array}{l}\text { Prospective, } 12 \text { months } \\
\text { follow up }\end{array}$ & 796 & & $\mathrm{MoCA}$ & $\begin{array}{c}\text { SBP, DBP } \\
\text { Ultra-short BPV } \\
\text { Daily BPV } \\
\text { Long-term BPV }\end{array}$ & $\begin{array}{c}\text { High and lower SBP } \rightarrow \text { Risk of } \\
\text { early PSCI } \uparrow\end{array}$ \\
\hline Levine, D.A. et al. [188] & $\begin{array}{l}\text { Cross section, } 90 \text { days } \\
\text { after stroke }\end{array}$ & 432 & \multirow{2}{*}{$\begin{array}{c}\text { Non-demented } \\
\text { No cognitive impairment }\end{array}$} & $\begin{array}{l}\text { Modified MMSE, Trails } \\
\text { A, and Trails B }\end{array}$ & SBP, DBP, PP, MAP & $\begin{array}{c}\text { Lower DBP } \rightarrow \text { Lower trails } \\
\text { B score }\end{array}$ \\
\hline Yamamoto, Y. et al. [189] & $\begin{array}{l}\text { Prospective, } 4.1 \text { years } \\
\text { follow up }\end{array}$ & 249 & & MMSE & Home BP (HBP) & $\uparrow \mathrm{HBP} \rightarrow$ Risk of late PSCI $\uparrow$ \\
\hline Sachdev, P.S., et al. [190] & $\begin{array}{l}\text { Cross section, 3-6 } \\
\text { months after stroke } \\
\text { Case control }\end{array}$ & $\begin{array}{l}169 \text { stroke } \\
103 \text { Control }\end{array}$ & & $\begin{array}{l}\text { Comprehensive } \\
\text { Neuropsychological } \\
\text { Assessment }\end{array}$ & $\begin{array}{l}\text { Diagnosis of } \\
\text { Hypertension }\end{array}$ & $\begin{array}{l}\text { Hypertension is not a risk } \\
\text { factor for PSCI }\end{array}$ \\
\hline $\begin{array}{l}\text { Ihle-Hansen, H. et al. } \\
\text { [191] }\end{array}$ & $\begin{array}{l}\text { Prospective, } 1 \text { ear } \\
\text { follow up }\end{array}$ & 166 & First-ever stroke & $\begin{array}{c}\text { Diagnosis of MCI } \\
\text { Diagnosis of dementia }\end{array}$ & SBP, DBP & $\begin{array}{c}\text { No association of BP level and } \\
\text { dementia or MCI }\end{array}$ \\
\hline Geng, S., et al. [193] & $\begin{array}{l}\text { Prospective, } 12 \text { months } \\
\text { follow up }\end{array}$ & 796 & & $\mathrm{MoCA}$ & $\begin{array}{c}\text { SBP, DBP } \\
\text { SBPV, DBPV }\end{array}$ & $\begin{array}{c}\uparrow \text { early SBPV } \rightarrow \text { Risk of late } \\
\text { PSCI } \uparrow\end{array}$ \\
\hline Lee, J.H. et al. [194] & $\begin{array}{l}\text { Prospective } 3 \text { months } \\
\text { follow up }\end{array}$ & 36 & Lacunar infarction & $\begin{array}{c}\text { MMSE, } \\
\text { Controlled Oral Word } \\
\text { Association Test, Digit } \\
\text { Symbol Coding test }\end{array}$ & DBPV, SBPV & $\begin{array}{c}\uparrow \text { early SBPV } \rightarrow \text { Risk of early } \\
\text { PSCI } \uparrow, \text { especially frontal } \\
\text { lobe dysfunction }\end{array}$ \\
\hline Kim, Y., et al. [195] & $\begin{array}{l}\text { Prospective, } 2.6 \text { years } \\
\text { follow up }\end{array}$ & 746 & & MMSE, MoCA & $\begin{array}{l}\text { SBP, DBP } \\
\text { SBPV, DBPV }\end{array}$ & $\uparrow \mathrm{BPV} \rightarrow$ Risk of late PSCI $\uparrow$ \\
\hline
\end{tabular}

$\uparrow$, increase; $\downarrow$. decrease; $\rightarrow$ leads to. 


\section{Management to Modify or Prevent PSCI}

\subsection{General Picture of BP Control in Stroke}

For the acute phase after a stroke, the patient usually experiences a period of elevated blood pressure due not only to autoregulation, which enhances cerebral perfusion to minimize the ischemic core, but also to acute stress, intracranial hypertension, and other systemic disarrangements [196]. In the acute phase, high BP was thought to be related to a worse clinical outcome in the Fukuoka study when it was $154 / 89 \mathrm{mmHg}$ [196], but a better outcome was found in another where BP was 150/70 mmHg [197]. A higher BP may interrupt the BBB, resulting in worsening brain edema, but a lower BP means a decrease in cerebral perfusion and an extension of the infarct size [197]. Regarding antihypertensive drug trials for acute stroke, early initiation of angiotensin converting enzyme inhibitors (ACEIs), angiotensin receptor blockers (ARBs), adrenoreceptor antagonist (both alpha and beta), and $C C B$ in the acute phase showed nearly neutral or negative findings for functional outcome or survival [198]. Recent American Heart Association (AHA) guidelines suggest that aggressive BP control may not be needed during the first $72 \mathrm{~h}$, unless comorbid systemic disorders warrant strict BP control, such as a hypertensive crisis causing organ damage, intravenous thrombolytic or endovascular therapy with successful recanalization, or a BP higher than 220/120 mmHg [199]. European Stroke Organization (ESO) guidelines suggested no aggressive control when BP is lower than $220 / 110 \mathrm{mmHg}$ in the first $24 \mathrm{~h}$ of stroke onset [200].

For secondary prevention, AHA guidelines suggest keeping office BP below 130/80 $\mathrm{mmHg}$ using thiazide, ACEI, and ARB [201].

\subsection{Evidence Regarding Modifying the Potential Effect of BP/BPV on PSCI}

Increased BPV or higher SBP may potentially raise the risk of PSCI, but the evidence regarding $\mathrm{BP} / \mathrm{BPV}$ control and PSCI prevention is inconclusive. In animal studies, angiotensin receptor blockers (ARBs) effectively prevented $A \beta 42$ toxicity, reactive microgliosis, and apoptotic cell death, leading to a reduced infarct size and prevention of PSCI following acute ischemic events within 30 days [202,203]. However, the human situation is more complicated. The appropriate time for medication seems to lead to controversial results. The Scandinavian Candesartan Acute Stroke Trial (SCAST) and the Valsartan Efficacy oN modesT blood pressUre Reduction in acute ischemic stroke (VENTURE) trial suggested early administration of ARBs may lead to worse functional outcomes or the risk of neurological deterioration [204,205]. Entering the subacute phase, ARBs were reported to be effective for preventing PSCI in one study [206] but not another [207]. Strict BP control ( $\leq 125 \mathrm{mmHg}$ vs. $\leq 140 \mathrm{mmHg}$ vs. $\leq 160 \mathrm{mmHg}$ ) also yielded no definite effect on the prevention of PSCI in the 1 year post-stroke period [191]. The Perindopril pROtection aGainst REcurrent Stroke Study (PROGRESS) showed a positive effect in cognitive function maintenance in a poststroke patient with a combination of ACEIs and indapamide that reduced the overall risk of PSCI by one-third [208]. In addition to ordinary antihypertensive medications, one study reported that tadalafil improved cerebral perfusion in patients with small vessel occlusion (SVO) [209]. However, there was no evaluation of cognition.

\subsection{Other Pharmacological and Nonpharmacological Approach to Modify or Prevent PSCI}

Statins showed a promising result in secondary stroke prevention, but the Heart Protection Study (HPS) and the PROspective Study of Pravastatin in the Elderly at Risk (PROSPER) showed that they had no positive impact on cognitive function [210,211]. One report suggested that a combination of piracetam and cinnarizine may improve late poststroke cognition in patients compared to those without treatment [212]. Cilostazol, an antiplatelet agent working on the P2Y12 receptor, is known for its antioxidative stress activity, neuroprotection (particularly toward hippocampal cells), and prevention of amyloid oligomerization $[213,214]$. One Japanese study demonstrated that it may facilitate cognitive recovery in the subacute phase beyond 1 month [215], but a study by a Korean group suggested it had no better effect on poststroke cognition compared to aspirin [216]. 
Acetylcholinesterase inhibitors are effective AD medications, and their efficacy was also tested in PSCI. Two randomized controlled trials focusing on late PSCI demonstrated significant cognitive recovery with these medications compared with a placebo $[217,218]$. Finally, a preliminary study showed a promising effect of amantadine on neurocognitive function [219].

Other than medication, there are some other methods to modify or prevent PSCI. Acupuncture was reported to be effective in preventing PSCI in retrospective studies, randomized control trials, and meta-analyses [220-222]. Physical training was said to be effective in cognitive improvement in one review but inconclusive in another $[223,224]$. Early cognitive training is effective for memory and attention [224], but the long-term effect is uncertain [18].

Diet is also important. Alcohol consumption is controversial in dementia patients: a light to moderate amount has a protective effect, but an excessive amount leads to direct neurotoxicity [225]. Alcohol consumption is also thought to be a risk factor for PSCI [71], and in PSD patients, it carried a higher risk of recurrent stroke [226]. On the other hand, coffee drinking, especially mocha, showed protective effects against poststroke dementia in subcortical infarction patients, but smoking may eliminate them [227]. The nutritional supplements cichotoline and L-acetyl carnitine were reported to have positive effects on improving poststroke cognitive performance and preventing poststroke cognitive impairment [18,228-230].

In the PSCI group, repetitive transcranial magnetic stimulation (rTMS) seemed to improve patients' activities of daily living (ADLs) and cognitive function by enhancing functional connectivity and the amplitude of low-frequency fluctuation in the bilateral mesial-prefrontal cortex, the cingulate gyrus, and many other brain regions [231,232]. Additionally, a systemic review found that excitatory or inhibitory stimulation of the dorsolateral prefrontal cortex (DLPFC) may improve patients' attention, working memory, long-term memory, and cognitive function [233], but there was a marked heterogeneity in the enrolled studies. Nevertheless, an in vivo study showed that low frequency rTMS may upregulate hippocampal neuron synaptic plasticity via the BDNF-TrkB pathway [234,235]. In the hippocampus, Calb2, Zic1, Kcnk9, and Grin3a genes were upregulated as were the glutamatergic synapses [236]. Furthermore, rTMS may have a beneficial effect on poststroke depression as well [237]. Music therapy (MT) may have a positive effect, but the data are conflicting. Some studies suggested a positive effect on verbal memory, focused attention, and quality of life in poststroke patients with PSCI, but others showed no improvement in mood or cognition $[238,239]$. The Figure 2 summarized the poststroke cognitive changes, factors affecting the cognitive function, along with possible interventions and treatments. 


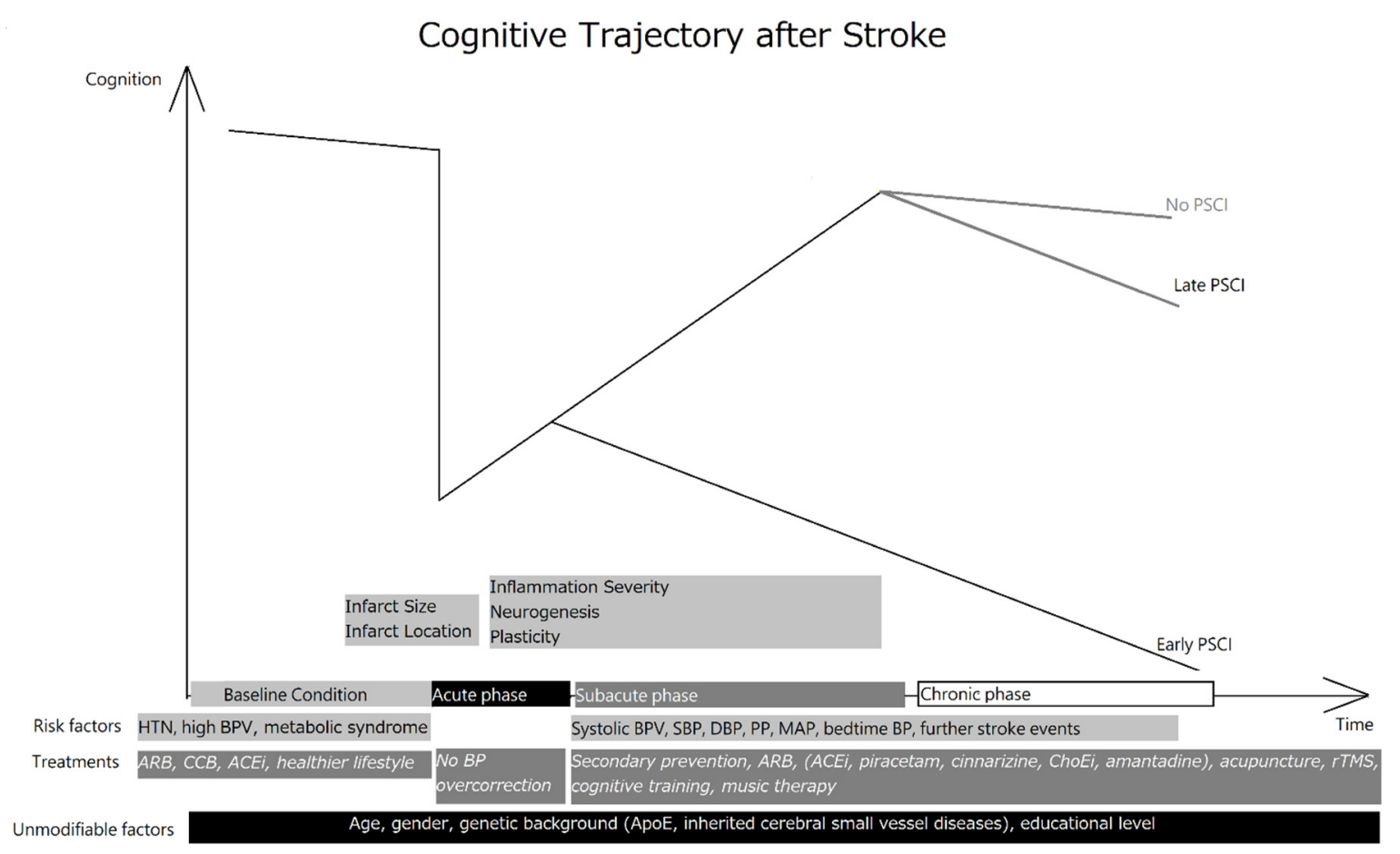

Figure 2. Cognitive trajectory after stroke.

\section{Limitation}

This review had several limitations. First, we enrolled mainly English-written studies, thus there was a certain degree of selection bias. Second, the studies and the reviews enrolled were highly heterogeneous. The definition of PSCI, its classification, diagnostic criteria, the parameters of BP, and the duration of follow up may have influenced the findings and the conclusions. Currently, a universally accepted consensus of this disease is still missing. Third, though we tried to figure out the underlying pathophysiology, there is a lack of human brain pathology or long-term functional imaging or pathology follow up to clarify the longitudinal change in a brain after a stroke.

\section{Conclusions}

Cognition alterations after a stroke are a complicated and dynamic process. Evidence suggests that a stroke may not only induce a cascade of inflammatory processes that interrupt circuits between cortical regions, leading to remote atrophic changes outside the lesion, but may also potentially interfere with the accumulation or the clearance of amyloids. The existence or the progression of AD pathology and SVD changes may contribute differently to early and late PSCI. The relationships between BP/BPV and PSCI/PSD are complicated and depend on particular parameters and different stages of stroke. BP or BPV may have a potential negative impact on the major pathophysiology of PSCI, including AD pathology, SVD changes, and ischemic neurodegeneration, and some evidence may suggest BP/BPV control for PSCI modification or prevention. Further studies may be warranted. A longitudinal study should be carried out that includes brain MRIs, functional imaging, and/or an amyloid or Tau PET to reveal the potential cerebral pathology interaction in PSCI patients. Large scale investigations into the definite effect of $\mathrm{BP} / \mathrm{BPV}$ changes at different stages of a stroke and appearance and progression of PSCI/PSD with both short-term and long-term cognitive outcomes may be considered as well.

Author Contributions: Conceptualization, P.-S.S., A.Y.W.C.; writing-original draft preparation, K.-P.L.; writing - review and editing, P.-S.S.; project administration, P.-S.S. All authors have read and agreed to the published version of the manuscript. 
Funding: This work was supported by National Cheng Kung University Hospital (Grant Number: NCKUH-11009022).

Institutional Review Board Statement: This study was approved by the Institutional Review Board of National Cheng Kung University Hospital (A-ER-109-429).

Informed Consent Statement: Not applicable.

Data Availability Statement: The data was available upon reasonable email request.

Acknowledgments: We thank the staff of the stroke center of National Cheng Kung University Hospital for their support.

Conflicts of Interest: The authors declare no conflict of interest.

\section{References}

1. Donnan, G.A.; Khatri, P. Stroke. Lancet 2008, 371, 1612-1623. [CrossRef]

2. Hu, H.H.; Sheng, W.Y.; Chu, F.L.; Lan, C.F.; Chiang, B.N. Incidence of stroke in Taiwan. Stroke 1992, 23, 1237-1241. [CrossRef]

3. Hu, H.H.; Chu, F.L.; Chiang, B.N.; Lan, C.F.; Sheng, W.Y.; Lo, Y.K.; Wong, W.J.; Luk, Y.O. Prevalence of stroke in Taiwan. Stroke 1989, 20, 858-863. [CrossRef]

4. Luengo-Fernandez, R.; Violato, M.; Candio, P.; Leal, J. Economic burden of stroke across Europe: A population-based cost analysis. Eur. Stroke J. 2020, 5, 17-25. [CrossRef]

5. Rajsic, S.; Gothe, H.; Borba, H.H.; Sroczynski, G.; Vujicic, J.; Toell, T.; Siebert, U. Economic burden of stroke: A systematic review on post-stroke care. Eur. J. Health Econ. 2019, 20, 107-134. [CrossRef] [PubMed]

6. Madureira, S.; Guerreiro, M.; Ferro, J.M. Dementia and cognitive impairment three months after stroke. Eur. J. Neurol. 2001, 8, 621-627. [CrossRef]

7. Pendlebury, S.T.; Rothwell, P.M. Prevalence, incidence, and factors associated with pre-stroke and post-stroke dementia: A systematic review and meta-analysis. Lancet Neurol. 2009, 8, 1006-1018. [CrossRef]

8. Lin, J.-H.; Lin, R.-T.; Tai, C.-T.; Hsieh, C.-L.; Hsiao, S.-F.; Liu, C.-K. Prediction of poststroke dementia. Neurology 2003, 61, 343-348. [CrossRef]

9. Jacova, C.; Pearce, L.; Costello, R.; McClure, L.A.; Holliday, S.L.; Hart, R.G.; Benavente, O.R.; Ms, L.A.P. Cognitive impairment in lacunar strokes: The SPS3 trial. Ann. Neurol. 2012, 72, 351-362. [CrossRef]

10. Makin, S.D.J.; Turpin, S.; Dennis, M.S.; Wardlaw, J. Cognitive impairment after lacunar stroke: Systematic review and metaanalysis of incidence, prevalence and comparison with other stroke subtypes. J. Neurol. Neurosurg. Psychiatry 2013, 84, 893-900. [CrossRef]

11. Qu, Y.; Zhuo, L.; Li, N.; Hu, Y.; Chen, W.; Zhou, Y.; Wang, J.; Tao, Q.; Hu, J.; Nie, X.; et al. Prevalence of Post-Stroke Cognitive Impairment in China: A Community-Based, Cross-Sectional Study. PLoS ONE 2015, 10, e0122864. [CrossRef]

12. Gao, C.-Y.; Lian, Y.; Zhang, M.; Zhang, L.-L.; Fang, C.-Q.; Deng, J.; Li, J.; Xu, Z.-Q.; Zhou, H.-D.; Wang, Y.-J. Association of dementia with death after ischemic stroke: A two-year prospective study. Exp. Ther. Med. 2016, 12, 1765-1769. [CrossRef]

13. Lugtmeijer, S.; Lammers, N.A.; de Haan, E.H.F.; de Leeuw, F.-E.; Kessels, R.P.C. Post-Stroke Working Memory Dysfunction: A Meta-Analysis and Systematic Review. Neuropsychol. Rev. 2021, 31, 202-219. [CrossRef]

14. Guo, J.; Wang, J.; Sun, W.; Liu, X. The advances of post-stroke depression: 2021 update. J. Neurol. 2021. [CrossRef]

15. Zhu, Y.; Zhao, S.; Fan, Z.; Li, Z.; He, F.; Lin, C.; Topatana, W.; Yan, Y.; Liu, Z.; Chen, Y.; et al. Evaluation of the Mini-Mental State Examination and the Montreal Cognitive Assessment for Predicting Post-stroke Cognitive Impairment During the Acute Phase in Chinese Minor Stroke Patients. Front. Aging Neurosci. 2020, 12, 236. [CrossRef] [PubMed]

16. Oh, H.; Park, J.; Seo, W. A 2-year prospective follow-up study of temporal changes associated with post-stroke cognitive impairment. Int. J. Nurs. Pract. 2018, 24, e12618. [CrossRef] [PubMed]

17. Mok, V.C.T.; Lam, B.Y.K.; Wong, A.; Ko, H.; Markus, H.S.; Wong, L.K.S. Early-onset and delayed-onset poststroke dementiarevisiting the mechanisms. Nat. Rev. Neurol. 2017, 13, 148-159. [CrossRef] [PubMed]

18. Mijajlović, M.D.; Pavlović, A.; Brainin, M.; Heiss, W.-D.; Quinn, T.J.; Ihle-Hansen, H.B.; Hermann, D.M.; Ben Assayag, E.; Richard, E.; Thiel, A.; et al. Post-stroke dementia-A comprehensive review. BMC Med. 2017, 15, 11. [CrossRef] [PubMed]

19. Morsund, Å.H.; Ellekjaer, H.; Gramstad, A.; Reiestad, M.T.; Midgard, R.; Sando, S.B.; Jonsbu, E.; Naess, H.; Ellekjær, H.; Næss, H. The development of cognitive and emotional impairment after a minor stroke: A longitudinal study. Acta Neurol. Scand. 2019, 140, 281-289. [CrossRef]

20. Alexandrova, M.L.; Danovska, M.P. Cognitive impairment one year after ischemic stroke: Predictorsand dynamics of significant determinants. Turk. J. Med. Sci. 2016, 46, 1366-1373. [CrossRef]

21. Wolf, T.J.; Barbee, A.R.; White, D. Executive Dysfunction Immediately after Mild Stroke. OTJR: Occup. Particip. Health 2011, 31, S23-S29. [CrossRef]

22. Aam, S.; Einstad, M.S.; Munthe-Kaas, R.; Lydersen, S.; Ihle-Hansen, H.; Knapskog, A.-B.; Ellekjær, H.; Seljeseth, Y.; Saltvedt, I. Post-stroke Cognitive Impairment-Impact of Follow-Up Time and Stroke Subtype on Severity and Cognitive Profile: The Nor-COAST Study. Front. Neurol. 2020, 11, 699. [CrossRef] [PubMed] 
23. Elgh, E.; Hu, X. Dynamic Trajectory of Long-Term Cognitive Improvement Up to 10 Years in Young Community-Dwelling Stroke Survivors: A Cohort Study. Front. Neurol. 2019, 10, 97. [CrossRef]

24. Sachdev, P.S.; Chen, X.; Brodaty, H.; Thompson, C.; Altendorf, A.; Wen, W. The determinants and longitudinal course of post-stroke mild cognitive impairment. J. Int. Neuropsychol. Soc. 2009, 15, 915-923. [CrossRef] [PubMed]

25. Mărgăritescu, O.; Mogoanta, L.; Pirici, I.; Pirici, D.; Cernea, D.; Mărgăritescu, C. Histopathological changes in acute ischemic stroke. Rom. J. Morphol. Embryol. 2009, 50, 327-339.

26. Kalaria, R.N.; Akinyemi, R.; Ihara, M. Stroke injury, cognitive impairment and vascular dementia. Biochim. Biophys. Acta (BBA) Mol. Basis Dis. 2016, 1862, 915-925. [CrossRef]

27. Lambertsen, K.L.; Biber, K.; Finsen, B. Inflammatory Cytokines in Experimental and Human Stroke. Br. J. Pharmacol. 2012, 32, 1677-1698. [CrossRef]

28. Swardfager, W.; Winer, D.A.; Herrmann, N.; Winer, S.; Lanctôt, K.L. Interleukin-17 in post-stroke neurodegeneration. Neurosci. Biobehav. Rev. 2013, 37, 436-447. [CrossRef] [PubMed]

29. Rothenburg, L.S.; Herrmann, N.; Swardfager, W.; Black, S.; Tennen, G.; Kiss, A.; Gladstone, D.; Ween, J.; Snaiderman, A.; Lanctot, K.L. The Relationship Between Inflammatory Markers and Post Stroke Cognitive Impairment. J. Geriatr. Psychiatry Neurol. 2010, 23, 199-205. [CrossRef] [PubMed]

30. Gulyaeva, N.V. Biochemical Mechanisms and Translational Relevance of Hippocampal Vulnerability to Distant Focal Brain Injury: The Price of Stress Response. Biochemostry 2019, 84, 1306-1328. [CrossRef] [PubMed]

31. Delattre, C.; Bournonville, C.; Auger, F.; Lopes, R.; Delmaire, C.; Henon, H.; Mendyk, A.M.; Bombois, S.; Devedjian, J.C.; Leys, D.; et al. Hippocampal Deformations and Entorhinal Cortex Atrophy as an Anatomical Signature of Long-Term Cognitive Impairment: From the MCAO Rat Model to the Stroke Patient. Transl. Stroke Res. 2018, 9, 294-305. [CrossRef] [PubMed]

32. Grysiewicz, R.; Gorelick, P.B. Key Neuroanatomical Structures for Post-Stroke Cognitive Impairment. Curr. Neurol. Neurosci. Rep. 2012, 12, 703-708. [CrossRef] [PubMed]

33. Thiel, A.; Cechetto, D.F.; Heiss, W.-D.; Hachinski, V.; Whitehead, S.N. Amyloid Burden, Neuroinflammation, and Links to Cognitive Decline After Ischemic Stroke. Stroke 2014, 45, 2825-2829. [CrossRef]

34. Cuartero, M.I.; García-Culebras, A.; Torres-López, C.; Medina, V.; Fraga, E.; Vázquez-Reyes, S.; Jareño-Flores, T.; García-Segura, J.M.; Lizasoain, I.; Moro, M.Á. Post-stroke Neurogenesis: Friend or Foe? Frontiers in cell and developmental biology. Front. Cell Dev. Biol. 2021, 9, 657846. [CrossRef] [PubMed]

35. Kempermann, G.; Song, H.; Gage, F.H. Neurogenesis in the Adult Hippocampus. Cold Spring Harb. Perspect. Biol. 2015,7 , a018812. [CrossRef]

36. Lindvall, O.; Kokaia, Z. Neurogenesis following Stroke Affecting the Adult Brain. Cold Spring Harb. Perspect. Biol. 2015, 7, a019034. [CrossRef]

37. Nakayama, H.; Jørgensen, H.S.; Raaschou, H.O.; Olsen, T.S. Recovery of upper extremity function in stroke patients: The Copenhagen stroke study. Arch. Phys. Med. Rehabil. 1994, 75, 394-398. [CrossRef]

38. Cuartero, M.; De La Parra, J.; Pérez-Ruiz, A.; Bravo-Ferrer, I.; Durán-Laforet, V.; Garcia-Culebras, A.; García-Segura, J.M.; Dhaliwal, J.; Frankland, P.W.; Lizasoain, I.; et al. Abolition of aberrant neurogenesis ameliorates cognitive impairment after stroke in mice. J. Clin. Investig. 2019, 129, 1536-1550. [CrossRef]

39. Khlif, M.S.; Werden, E.; Egorova, N.; Boccardi, M.; Redolfi, A.; Bird, L.; Brodtmann, A. Assessment of longitudinal hippocampal atrophy in the first year after ischemic stroke using automatic segmentation techniques. NeuroImage Clin. 2019, $24,102008$. [CrossRef]

40. Brodtmann, A.; Khlif, M.S.; Egorova, N.; Veldsman, M.; Bird, L.J.; Werden, E. Dynamic Regional Brain Atrophy Rates in the First Year After Ischemic Stroke. Stroke 2020, 51, e183-e192. [CrossRef]

41. Schaapsmeerders, P.; Tuladhar, A.M.; Maaijwee, N.A.M.; Rutten-Jacobs, L.C.A.; Arntz, R.M.; Schoonderwaldt, H.C.; Dorresteijn, L.D.A.; Van Dijk, E.J.; Kessels, R.P.C.; De Leeuw, F.-E. Lower Ipsilateral Hippocampal Integrity after Ischemic Stroke in Young Adults: A Long-Term Follow-Up Study. PLoS ONE 2015, 10, e0139772. [CrossRef] [PubMed]

42. Ter Telgte, A.; Van Leijsen, E.M.C.; Wiegertjes, K.; Klijn, C.; Tuladhar, A.M.; De Leeuw, F.-E. Cerebral small vessel disease: From a focal to a global perspective. Nat. Rev. Neurol. 2018, 14, 387-398. [CrossRef]

43. Kim, Y.J.; Kwon, H.K.; Lee, J.M.; Cho, H.; Kim, H.J.; Park, H.K.; Jung, N.; Lee, J.S.; Lee, J.; Jang, Y.K. Gray and white matter changes linking cerebral small vessel disease to gait disturbances. Neurology 2016, 86, 1199-1207. [CrossRef]

44. Staals, J.; Makin, S.; Doubal, F.N.; Dennis, M.S.; Wardlaw, J.M. Stroke subtype, vascular risk factors, and total MRI brain small-vessel disease burden. Neurology 2014, 83, 1228-1234. [CrossRef] [PubMed]

45. Duering, M.; Righart, R.; Csanadi, E.; Jouvent, E.; Hervé, D.; Chabriat, H.; Dichgans, M. Incident subcortical infarcts induce focal thinning in connected cortical regions. Neurology 2012, 79, 2025-2028. [CrossRef]

46. Peng, C.-Y.; Chen, Y.-C.; Cui, Y.; Zhao, D.-L.; Jiao, Y.; Tang, T.-Y.; Ju, S.; Teng, G.-J. Regional Coherence Alterations Revealed by Resting-State fMRI in Post-Stroke Patients with Cognitive Dysfunction. PLoS ONE 2016, 11, e0159574. [CrossRef]

47. Tuladhar, A.M.; Snaphaan, L.; Shumskaya, E.; Rijpkema, M.; Fernandez, G.; Norris, D.G.; De Leeuw, F.-E. Default Mode Network Connectivity in Stroke Patients. PLoS ONE 2013, 8, e66556. [CrossRef]

48. Liu, J.; Wang, Q.; Liu, F.; Song, H.; Liang, X.; Lin, Z.; Hong, W.; Yang, S.; Huang, J.; Zheng, G.; et al. Altered functional connectivity in patients with post-stroke memory impairment: A resting fMRI study. Exp. Ther. Med. 2017, 14, 1919-1928. [CrossRef] 
49. Aguayo, R.D.; Grana, M.; Fernández-Andújar, M.; López-Cancio, E.; Cáceres, C.; Bargalló, N.; Barrios, M.; Clemente, I.; Monserrat, P.T.; Sas, M.A.; et al. Structural Integrity of the Contralesional Hemisphere Predicts Cognitive Impairment in Ischemic Stroke at Three Months. PLoS ONE 2014, 9, e86119.

50. Vinciguerra, L.; Lanza, G.; Puglisi, V.; Fisicaro, F.; Pennisi, M.; Bella, R.; Cantone, M. Update on the Neurobiology of Vascular Cognitive Impairment: From Lab to Clinic. Int. J. Mol. Sci. 2020, 8, 2977. [CrossRef]

51. Li, J.; Zhao, Y.; Mao, J. Association between the extent of white matter damage and early cognitive impairment following acute ischemic stroke. Exp. Ther. Med. 2017, 13, 909-912. [CrossRef]

52. Mok, V.C.; Liu, W.Y.; Wong, A. Detection of amyloid plaques in patients with post-stroke dementia. Hong Kong Med. J. 2016, 22, S40-S42. [PubMed]

53. Akinyemi, R.O.; Allan, L.M.; Oakley, A.; Kalaria, R.N. Hippocampal Neurodegenerative Pathology in Post-stroke Dementia Compared to Other Dementias and Aging Controls. Front. Neurosci. 2017, 11, 717. [CrossRef]

54. Jokinen, H.; Kalska, H.; Mäntylä, R.; Ylikoski, R.; Hietanen, M.; Pohjasvaara, T.; Kaste, M.; Erkinjuntti, T. White matter hyperintensities as a predictor of neuropsychological deficits post-stroke. J. Neurol. Neurosurg. Psychiatry 2005, 76, 1229-1233. [CrossRef]

55. Wollenweber, F.A.; Därr, S.; Müller, C.; Duering, M.; Buerger, K.; Zietemann, V.; Malik, R.; Brendel, M.; Ertl-Wagner, B.; Bartenstein, P.; et al. Prevalence of Amyloid Positron Emission Tomographic Positivity in Poststroke Mild Cognitive Impairment. Stroke 2016, 47, 2645-2648. [CrossRef]

56. Del Brutto, O.H.; Mera, R.M.; Zambrano, M.; Lama, J. Cognitive performance in community-dwelling older adults with stroke and the contribution of age and diffuse subcortical damage: A population-based study in rural Ecuador (The Atahualpa Project). Aging Clin. Exp. Res. 2015, 27, 647-652. [CrossRef] [PubMed]

57. Casolla, B.; Caparros, F.; Cordonnier, C.; Bombois, S.; Hénon, H.; Bordet, R.; Orzi, F.; Leys, D. Biological and imaging predictors of cognitive impairment after stroke: A systematic review. J. Neurol. 2019, 266, 2593-2604. [CrossRef]

58. Kliper, E.; Ben Assayag, E.; Tarrasch, R.; Artzi, M.; Korczyn, A.D.; Shenhar-Tsarfaty, S.; Aizenstein, O.; Hallevi, H.; Mike, A.; Shopin, L.; et al. Cognitive State following Stroke: The Predominant Role of Preexisting White Matter Lesions. PLoS ONE 2014, 9 , e105461. [CrossRef]

59. Kim, B.J.; Oh, M.-Y.; Jang, M.S.; Han, M.-K.; Lee, J.; Lee, J.; Kang, Y.; Yu, K.-H.; Lee, B.-C.; Kim, S.; et al. Medial Temporal Atrophy and Memory Dysfunction in Poststroke Cognitive Impairment-No Dementia. J. Clin. Neurol. 2012, 8, 43-50. [CrossRef] [PubMed]

60. Molad, J.; Hallevi, H.; Korczyn, A.D.; Kliper, E.; Auriel, E.; Bornstein, N.M.; Ben Assayag, E. Vascular and Neurodegenerative Markers for the Prediction of Post-Stroke Cognitive Impairment: Results from the TABASCO Study. J. Alzheimer's Dis. 2019, 70, 889-898. [CrossRef]

61. Firbank, M.J.; Burton, E.J.; Barber, R.; Stephens, S.; Kenny, R.A.; Ballard, C.; Kalaria, R.N.; O’Brien, J. Medial temporal atrophy rather than white matter hyperintensities predict cognitive decline in stroke survivors. Neurobiol. Aging 2007, 28, 1664-1669. [CrossRef]

62. Yang, J.; Wong, A.; Wang, Z.; Liu, W.; Au, L.; Xiong, Y.; Chu, W.W.; Leung, E.Y.; Chen, S.; Lau, C.; et al. Risk factors for incident dementia after stroke and transient ischemic attack. Alzheimer's Dement. 2015, 11, 16-23. [CrossRef]

63. Christ, N.; Mocke, V.; Fluri, F. Cerebral microbleeds are associated with cognitive decline early after ischemic stroke. J. Neurol. 2019, 266, 1091-1094. [CrossRef] [PubMed]

64. Mok, V.C.T.; Lam, B.Y.K.; Wang, Z.; Liu, W.; Au, L.; Leung, E.Y.L.; Chen, S.; Yang, J.; Chu, W.C.W.; Lau, A.Y.L.; et al. Delayed-onset dementia after stroke or transient ischemic attack. Alzheimers Dement 2016, 12, 1167-1176. [CrossRef] [PubMed]

65. Mok, V.; Xiong, Y.; Wong, K.K.; Wong, A.; Schmidt, R.; Chu, W.W.; Hu, X.; Leung, E.Y.L.; Chen, S.; Chen, Y.; et al. Predictors for cognitive decline in patients with confluent white matter hyperintensities. Alzheimer's Dement. 2012, 8, S96-S103. [CrossRef] [PubMed]

66. Dufouil, C.; Godin, O.; Chalmers, J.; Coskun, O.; MacMahon, S.; Tzourio-Mazoyer, N.; Bousser, M.-G.; Anderson, C.; Mazoyer, B.; Tzourio, C. Severe Cerebral White Matter Hyperintensities Predict Severe Cognitive Decline in Patients with Cerebrovascular Disease History. Stroke 2009, 40, 2219-2221. [CrossRef]

67. Liu, W.; Wong, A.; Au, L.; Yang, J.; Wang, Z.; Leung, E.Y.L.; Chen, S.; Ho, C.L.; Mok, V.C.T. Influence of Amyloid- $\beta$ on Cognitive Decline After Stroke/Transient Ischemic Attack: Three-Year Longitudinal Study. Stroke 2015, 46, 3074-3080. [CrossRef]

68. Gao, Q.; Fan, Y.; Mu, L.-Y.; Ma, L.; Song, Z.-Q.; Zhang, Y.-N. S100B and ADMA in cerebral small vessel disease and cognitive dysfunction. J. Neurol. Sci. 2015, 354, 27-32. [CrossRef]

69. Castellazzi, M.; Trentini, A.; Romani, A.; Valacchi, G.; Bellini, T.; Bonaccorsi, G.; Fainardi, E.; Cavicchio, C.; Passaro, A.; Zuliani, G.; et al. Decreased arylesterase activity of paraoxonase-1 (PON-1) might be a common denominator of neuroinflammatory and neurodegenerative diseases. Int. J. Biochem. Cell Biol. 2016, 81, 356-363. [CrossRef]

70. Fleszar, M.G.; Wiśniewski, J.; Zboch, M.; Diakowska, D.; Gamian, A.; Krzystek-Korpacka, M. Targeted metabolomic analysis of nitric oxide/L-arginine pathway metabolites in dementia: Association with pathology, severity, and structural brain changes. Sci. Rep. 2019, 9, 13764. [CrossRef]

71. Wu, J.-X.; Xue, J.; Zhuang, L.; Liu, C.-F. Plasma parameters and risk factors of patients with post-stroke cognitive impairment. Ann. Palliat. Med. 2020, 9, 45-52. [CrossRef] 
72. Prodjohardjono, A.; Vidyanti, A.N.; Susianti, N.A.; Sudarmanta; Sutarni, S.; Setyopranoto, I. Higher level of acute serum VEGF and larger infarct volume are more frequently associated with post-stroke cognitive impairment. PLoS ONE 2020, 15, e0239370. [CrossRef]

73. Liu, M.; Zhou, K.; Li, H.; Dong, X.; Tan, G.; Chai, Y.; Wang, W.; Bi, X. Potential of serum metabolites for diagnosing post-stroke cognitive impairment. Mol. BioSyst. 2015, 11, 3287-3296. [CrossRef]

74. Chi, N.-F.; Chao, S.-P.; Huang, L.-K.; Chan, L.; Chen, Y.-R.; Chiou, H.-Y.; Hu, C.-J. Plasma Amyloid Beta and Tau Levels Are Predictors of Post-stroke Cognitive Impairment: A Longitudinal Study. Front. Neurol. 2019, 10, 715. [CrossRef]

75. Liu, Y.; Chen, H.; Zhao, K.; He, W.; Lin, S.; He, J. High levels of plasma fibrinogen are related to post-stroke cognitive impairment. Brain Behav. 2019, 9, e01391. [CrossRef]

76. Guo, J.; Su, W.; Fang, J.; Chen, N.; Zhou, M.; Zhang, Y.; He, L. Elevated CRP at admission predicts post-stroke cognitive impairment in Han Chinese patients with intracranial arterial stenosis. Neurol. Res. 2018, 40, 292-296. [CrossRef]

77. Ben Assayag, E.; Tene, O.; Korczyn, A.; Shopin, L.; Auriel, E.; Molad, J.; Hallevi, H.; Kirschbaum, C.; Bornstein, N.; ShenharTsarfaty, S.; et al. High hair cortisol concentrations predict worse cognitive outcome after stroke: Results from the TABASCO prospective cohort study. Psychoneuroendocrinology 2017, 82, 133-139. [CrossRef]

78. Ran, F.; Liu, F.; Zhang, Y.; Chen, L. Serum Uric Acid and High-Sensitivity C-Reactive Protein as Predictors of Cognitive Impairment in Patients with Cerebral Infarction. Dement. Geriatr. Cogn. Disord. 2020, 49, 235-242. [CrossRef]

79. Pascoe, M.C.; Linden, T. Folate and MMA predict cognitive impairment in elderly stroke survivors: A cross sectional study. Psychiatry Res. 2016, 243, 49-52. [CrossRef]

80. Yang, Z.; Wang, H.; Edwards, D.; Ding, C.; Yan, L.; Brayne, C.; Mant, J. Association of blood lipids, atherosclerosis and statin use with dementia and cognitive impairment after stroke: A systematic review and meta-analysis. Ageing Res. Rev. 2020, 57, 100962. [CrossRef]

81. Zhu, Z.; Zhong, C.; Guo, D.; Bu, X.; Xu, T.; Guo, L.; Liu, J.; Zhang, J.; Li, D.; Zhang, J.; et al. Multiple biomarkers covering several pathways improve predictive ability for cognitive impairment among ischemic stroke patients with elevated blood pressure. Atherosclerosis 2019, 287, 30-37. [CrossRef]

82. Li, P.; Quan, W.; Lu, D.; Wang, Y.; Zhang, H.-H.; Liu, S.; Jiang, R.-C.; Zhou, Y.-Y. Association between Metabolic Syndrome and Cognitive Impairment after Acute Ischemic Stroke: A Cross-Sectional Study in a Chinese Population. PLoS ONE 2016, 11, e0167327. [CrossRef] [PubMed]

83. Tong, Z.; Wang, W.; Luo, W.; Lv, J.; Li, H.; Luo, H.; Jia, J.; He, R. Urine Formaldehyde Predicts Cognitive Impairment in Post-Stroke Dementia and Alzheimer's Disease. J. Alzheimer's Dis. 2016, 55, 1031-1038. [CrossRef]

84. Zhang, X.; Bi, X. Post-Stroke Cognitive Impairment: A Review Focusing on Molecular Biomarkers. J. Mol. Neurosci. 2020, 70, 1244-1254. [CrossRef]

85. Wagle, J.; Farner, L.; Flekkøy, K.; Wyller, T.B.; Sandvik, L.; Eiklid, K.L.; Fure, B.; Stensrød, B.; Engedal, K. Cognitive impairment and the role of the ApoE epsilon4-allele after stroke-A 13 months follow-up study. Int. J. Geriatr. Psychiatry 2010, 25, 833-842. [CrossRef]

86. Wagle, J.; Farner, L.; Flekkøy, K.; Wyller, T.B.; Sandvik, L.; Eiklid, K.L.; Fure, B.; Stensrød, B.; Engedal, K. Association between ApoE epsilon4 and cognitive impairment after stroke. Dement Geriatr. Cogn. Disord. 2009, 27, 525-533. [CrossRef]

87. Rezaei, S.; Mobarake, K.A.; Saberi, A. BDNF (rs6265) Val <Met polymorphism can buffer cognitive functions against post stroke CT/MRI pathological findings. Appl. Neuropsychol. Adult 2020, 2, 1-12.

88. Pendlebury, S.T.; Poole, D.; Burgess, A.; Duerden, J. APOE- $\varepsilon 4$ Genotype and Dementia Before and After Transient Ischemic Attack and Stroke: Population-Based Cohort Study. Stroke 2020, 51, 751-758. [CrossRef]

89. Donnellan, C.; Redha, N.; Al Banna, M.; Al Sharoqi, I.; Al-Jishi, A.; Bakhiet, M.; Taha, S.; Abdulla, F.; Walsh, P. Associations between ApoE gene and psychological consequences post stroke in a Bahraini cohort. Asian J. Psychiatry 2019, 39, 135-142. [CrossRef]

90. Pohjasvaara, T.; Mäntylä, R.; Salonen, O.; Aronen, H.J.; Ylikoski, R.; Hietanen, M.; Kaste, M.; Erkinjuntti, T. How Complex Interactions of Ischemic Brain Infarcts, White Matter Lesions, and Atrophy Relate to Poststroke Dementia. Arch. Neurol. 2000, 57, 1295-1300. [CrossRef]

91. Béjot, Y.; Aboa-Eboule, C.; Durier, J.; Rouaud, O.; Agnes; Ponavoy, J.E.; Richard, D.; Moreau, T.; Giroud, M. Prevalence of early dementia after first-ever stroke: A 24-year population-based study. Stroke 2011, 42, 607-612. [CrossRef]

92. Ding, X.; Li, C.-Y.; Wang, Q.-S.; Du, F.-Z.; Ke, Z.-W.; Peng, F.; Wang, J.; Chen, L. Patterns in default-mode network connectivity for determining outcomes in cognitive function in acute stroke patients. Neuroscience 2014, 277, 637-646. [CrossRef]

93. Hagberg, G.; Ihle-Hansen, H.; Fure, B.; Thommessen, B.; Ihle-Hansen, H.; Øksengård, A.R.; Beyer, M.K.; Wyller, T.B.; Müller, E.G.; Pendlebury, S.T.; et al. No evidence for amyloid pathology as a key mediator of neurodegeneration post-stroke-A seven-year follow-up study. BMC Neurol. 2020, 20, 174. [CrossRef]

94. Wollenweber, F.A.; Zietemann, V.; Rominger, A.; Opherk, C.; Bayer-Karpinska, A.; Gschwendtner, A.; Andrews, L.C.; Bürger, K.; Duering, M.; Dichgans, M. The Determinants of Dementia after Stroke (DEDEMAS) Study: Protocol and Pilot Data. Int. J. Stroke 2013, 9, 387-392. [CrossRef]

95. Huang, K.-L.; Hsiao, I.-T.; Ho, M.-Y.; Hsu, J.-L.; Chang, Y.-J.; Chang, T.-Y.; Liu, C.-H.; Chang, C.-H.; Wu, Y.-M.; Wu, K.-Y.; et al. Investigation of reactive astrogliosis effect on post-stroke cognitive impairment. J. Neuroinflammation 2020, 17, 308. [CrossRef] 
96. Rothwell, P.M.; Howard, S.C.; Dolan, E.; O’Brien, E.; Dobson, J.; Dahlöf, B.; Sever, P.S.; Poulter, N.R. Prognostic significance of visit-to-visit variability, maximum systolic blood pressure, and episodic hypertension. Lancet 2010, 375, 895-905. [CrossRef]

97. Yu, J.-M.; Kong, Q.-Y.; Schoenhagen, P.; Shen, T.; He, Y.-S.; Wang, J.-W.; Zhao, Y.-P.; Shi, D.-N.; Zhong, B.-L. The prognostic value of long-term visit-to-visit blood pressure variability on stroke in real-world practice: A dynamic cohort study in a large representative sample of Chinese hypertensive population. Int. J. Cardiol. 2014, 177, 995-1000. [CrossRef] [PubMed]

98. Lau, K.-K.; Wong, Y.-K.; Teo, K.-C.; Chang, R.S.; Chan, K.-H.; Hon, S.F.; Wat, K.-L.; Cheung, R.T.; Li, L.S.; Siu, C.-W.; et al. Long-Term Prognostic Implications of Visit-to-Visit Blood Pressure Variability in Patients with Ischemic Stroke. Am. J. Hypertens. 2014, 27, 1486-1494. [CrossRef] [PubMed]

99. Lau, K.K.; Wong, Y.K.; Chang, R.S.K.; Teo, K.C.; Hon, S.F.K.; Chan, K.H.; Wat, K.L.; Cheung, R.T.F.; Li, L.S.W.; Siu, C.W.; et al. Visit-to-visit systolic blood pressure variability predicts all-cause and cardiovascular mortality after lacunar infarct. Eur. J. Neurol. 2013, 21, 319-325. [CrossRef] [PubMed]

100. Launer, L.J.; Ross, G.W.; Petrovitch, H.; Masaki, K.; Foley, D.; White, L.R.; Havlik, R.J. Midlife blood pressure and dementia: The Honolulu-Asia aging study. Neurobiol. Aging 2000, 21, 49-55. [CrossRef]

101. Kivipelto, M.; Helkala, E.-L.; Laakso, M.; Hänninen, T.; Hallikainen, M.; Alhainen, K.; Soininen, H.; Tuomilehto, J.; Nissinen, A. Midlife vascular risk factors and Alzheimer's disease in later life: Longitudinal, population based study. BMJ 2001, 322, 1447-1451. [CrossRef]

102. Yamada, M.; Kasagi, F.; Sasaki, H.; Masunari, N.; Mimori, Y.; Suzuki, G. Association Between Dementia and Midlife Risk Factors: The Radiation Effects Research Foundation Adult Health Study. J. Am. Geriatr. Soc. 2003, 51, 410-414. [CrossRef] [PubMed]

103. Whitmer, R.A.; Sidney, S.; Selby, J.; Johnston, S.C.; Yaffe, K. Midlife cardiovascular risk factors and risk of dementia in late life. Neurology 2005, 64, 277-281. [CrossRef]

104. Yoshitake, T.; Kiyohara, Y.; Kato, I.; Ohmura, T.; Iwamoto, H.; Nakayama, K.; Ohmori, S.; Nomiyama, K.; Kawano, H.; Ueda, K.; et al. Incidence and risk factors of vascular dementia and Alzheimer's disease in a defined elderly Japanese population: The Hisayama Study. Neurology 1995, 45, 1161-1168. [CrossRef] [PubMed]

105. Qin, H.; Zhu, B.; Hu, C.; Zhao, X. Later-Onset Hypertension Is Associated with Higher Risk of Dementia in Mild Cognitive Impairment. Front. Neurol. 2020, 11, 557977. [CrossRef] [PubMed]

106. Zúñiga-Salazar, G.A.; Hincapié-Arias, S.M.; Salazar-Bolaños, E.E.; Lara-Terán, J.J.; Cáceres-Vinueza, S.V.; Duarte-Vera, Y.C. Impact of arterial hypertension on the cognitive function of patients between 45 and 65 years. Luis Vernaza Hospital, Guayaquil, Ecuador. Arch. Cardiol. Mex. 2020, 90, 284-292. [PubMed]

107. Bahchevanov, K.M.; Dzhambov, A.M.; Chompalov, K.; Massaldjieva, R.; Atanassova, P.; Mitkov, M.D. Contribution of Components of Metabolic Syndrome to Cognitive Performance in Middle-Aged Adults. Arch. Clin. Neuropsychol. 2021, 36, 498-506. [CrossRef] [PubMed]

108. Boo, Y.Y.; Jutila, O.-E.; Cupp, M.A.; Manikam, L.; Cho, S.-I. The identification of established modifiable mid-life risk factors for cardiovascular disease which contribute to cognitive decline: Korean Longitudinal Study of Aging (KLoSA). Aging Clin. Exp. Res. 2021. [CrossRef]

109. Sun, D.; Thomas, E.A.; Launer, L.J.; Sidney, S.; Yaffe, K.; Fornage, M. Association of blood pressure with cognitive function at midlife: A Mendelian randomization study. BMC Med. Genom. 2020, 13, 121. [CrossRef] [PubMed]

110. Shim, Y.S.; Shin, H.-E. Impact of the ambulatory blood pressure monitoring profile on cognitive and imaging findings of cerebral small-vessel disease in older adults with cognitive complaints. J. Hum. Hypertens. 2021. [CrossRef]

111. Antonazzo, B.; Marano, G.; Romagnoli, E.; Ronzoni, S.; Frati, G.; Sani, G.; Janiri, L.; Mazza, M. Impact of arterial hypertension and its management strategies on cognitive function and dementia: A comprehensive umbrella review. Minerva Cardioangiol 2020. [CrossRef]

112. Kuller, L.H.; Lopez, O.L.; Newman, A.B.; Beauchamp, N.J.; Burke, G.; Dulberg, C.; Fitzpatrick, A.; Fried, L.; Haan, M.N. Risk Factors for Dementia in the Cardiovascular Health Cognition Study. Neuroepidemiology 2003, 22, 13-22. [CrossRef] [PubMed]

113. Tyas, S.L.; Manfreda, J.; Strain, L.; Montgomery, P.R. Risk factors for Alzheimer's disease: A population-based, longitudinal study in Manitoba, Canada. Int. J. Epidemiol. 2001, 30, 590-597. [CrossRef]

114. Carmona-Abellan, M.; Trzeciak, M.; Fernández, M.; Echeveste, B.; Imaz, L.; Luquin, M.-R.; Riverol, M. Blood Pressure and Risk of Cognitive Impairment: The Role of Vascular Disease in Neurodegeneration. Brain Sci. 2021, 11, 385. [CrossRef]

115. Hestad, K.; Engedal, K.; Schirmer, H.; Strand, B.H. The Effect of Blood Pressure on Cognitive Performance. An 8-Year Follow-Up of the Tromsø Study, Comprising People Aged 45-74 Years. Front. Psychol. 2020, 11, 607. [CrossRef]

116. Shang, S.; Li, P.; Deng, M.; Jiang, Y.; Chen, C.; Qu, Q. The Age-Dependent Relationship between Blood Pressure and Cognitive Impairment: A Cross-Sectional Study in a Rural Area of Xi'an, China. PLoS ONE 2016, 11, e0159485. [CrossRef]

117. Feng, R.; Rolls, E.T.; Cheng, W.; Feng, J. Hypertension is associated with reduced hippocampal connectivity and impaired memory. EBioMedicine 2020, 61, 103082. [CrossRef]

118. Li, H.; Sun, D.; Lu, D.; Zhang, J.; Zeng, J. Low Hippocampal Dentate Gyrus Volume Associated with Hypertension-Related Cognitive Impairment. Am. J. Alzheimer's Dis. Other Dementiasr 2020, 35, 1533317520949782.

119. Triantafyllou, A.; Ferreira, J.P.; Kobayashi, M.; Micard, E.; Xie, Y.; Kearney-Schwartz, A.; Hossu, G.; Rossignol, P.; Bracard, S.; Benetos, A. Longer Duration of Hypertension and MRI Microvascular Brain Alterations Are Associated with Lower Hippocampal Volumes in Older Individuals with Hypertension. J. Alzheimer's Dis. 2020, 74, 227-235. [CrossRef] [PubMed] 
120. Lane, C.; Barnes, J.; Nicholas, J.M.; Sudre, C.; Cash, D.M.; Parker, T.D.; Malone, I.B.; Lu, K.; James, S.-N.; Keshavan, A.; et al. Associations between blood pressure across adulthood and late-life brain structure and pathology in the neuroscience substudy of the 1946 British birth cohort (Insight 46): An epidemiological study. Lancet Neurol. 2019, 18, 942-952. [CrossRef]

121. Mok, V.; Kim, J.S. Prevention and Management of Cerebral Small Vessel Disease. J. Stroke 2015, 17, 111-122. [CrossRef] [PubMed]

122. Liu, Y.; Dong, Y.-H.; Lyu, P.-Y.; Chen, W.-H.; Li, R. Hypertension-Induced Cerebral Small Vessel Disease Leading to Cognitive Impairment. Chin. Med. J. 2018, 131, 615-619. [CrossRef] [PubMed]

123. Hawkins, K.A.; Emadi, N.; Pearlson, G.D.; Winkler, A.M.; Taylor, B.; Dulipsingh, L.; King, D.; Pittman, B.; Blank, K. Hyperinsulinemia and elevated systolic blood pressure independently predict white matter hyperintensities with associated cognitive decrement in the middle-aged offspring of dementia patients. Metab. Brain Dis. 2017, 32, 849-857. [CrossRef] [PubMed]

124. Zhou, L.W.; Panenka, W.J.; Al-Momen, G.; Gicas, K.M.; Thornton, A.E.; Jones, A.A.; Woodward, M.; Heran, M.K.S.; Vertinsky, A.T.; Su, W.; et al. Cerebral Small Vessel Disease, Risk Factors, and Cognition in Tenants of Precarious Housing. Stroke 2020, 51, 3271-3278. [CrossRef]

125. Mortensen, K.N.; Sanggaard, S.; Mestre, H.; Lee, H.; Kostrikov, S.; Xavier, A.L.; Gjedde, A.; Benveniste, H.; Nedergaard, M. Impaired Glymphatic Transport in Spontaneously Hypertensive Rats. J. Neurosci. 2019, 39, 6365-6377. [CrossRef]

126. Held, F.; Morris, A.W.; Pirici, D.; Niklass, S.; Sharp, M.M.; Garz, C.; Assmann, A.; Heinze, H.-J.; Schreiber, F.; Carare, R.O.; et al. Vascular basement membrane alterations and $\beta$-amyloid accumulations in an animal model of cerebral small vessel disease. Clin. Sci. 2017, 131, 1001-1013. [CrossRef]

127. Bender, A.R.; Raz, N. Age-related differences in memory and executive functions in healthy APOE $\varepsilon 4$ carriers: The contribution of individual differences in prefrontal volumes and systolic blood pressure. Neuropsychologia 2012, 50, 704-714. [CrossRef]

128. Presa, J.L.; Saravia, F.; Bagi, Z.; Filosa, J.A. Vasculo-Neuronal Coupling and Neurovascular Coupling at the Neurovascular Unit: Impact of Hypertension. Front. Physiol. 2020, 11, 584135. [CrossRef]

129. Nilsson, P.M. Early Vascular Aging in Hypertension. Front. Cardiovasc. Med. 2020, 7, 6. [CrossRef]

130. Iulita, M.F.; De La Colina, A.N.; Girouard, H. Arterial stiffness, cognitive impairment and dementia: Confounding factor or real risk? J. Neurochem. 2018, 144, 527-548. [CrossRef]

131. MacIntosh, B.J.; Shirzadi, Z.; Atwi, S.; Detre, J.A.; Dolui, S.; Bryan, R.N.; Launer, L.J.; Swardfager, W. Metabolic and vascular risk factors are associated with reduced cerebral blood flow and poorer midlife memory performance. Hum. Brain Mapp. 2019, 41, 855-864. [CrossRef] [PubMed]

132. Iadecola, C. The Neurovascular Unit Coming of Age: A Journey through Neurovascular Coupling in Health and Disease. Neuron 2017, 96, 17-42. [CrossRef] [PubMed]

133. de Montgolfier, O.; Pouliot, P.; Gillis, M.; Ferland, G.; Lesage, F.; Thorin-Trescases, N.; Thorin, É. Systolic hypertension-induced neurovascular unit disruption magnifies vascular cognitive impairment in middle-age atherosclerotic $\operatorname{LDLr}(-/-): \mathrm{hApoB}(+/+)$ mice. Geroscience 2019, 41, 511-532. [CrossRef]

134. de Montgolfier, O. High Systolic Blood Pressure Induces Cerebral Microvascular Endothelial Dysfunction, Neurovascular Unit Damage, and Cognitive Decline in Mice. Hypertension 2019, 73, 217-228. [CrossRef] [PubMed]

135. Iadecola, C. The Pathobiology of Vascular Dementia. Neuron 2013, 80, 844-866. [CrossRef] [PubMed]

136. Rosenberg, G.A. Extracellular matrix inflammation in vascular cognitive impairment and dementia. Clin. Sci. 2017, 131, 425-437. [CrossRef] [PubMed]

137. Pantoni, L. Cerebral small vessel disease: From pathogenesis and clinical characteristics to therapeutic challenges. Lancet Neurol. 2010, 9, 689-701. [CrossRef]

138. Katsi, V.; Marketou, M.; Maragkoudakis, S.; Didagelos, M.; Charalambous, G.; Parthenakis, F.; Tsioufis, C.; Tousoulis, D. Blood-brain barrier dysfunction: The undervalued frontier of hypertension. J. Hum. Hypertens. 2020, 34, 682-691. [CrossRef]

139. Yamazaki, Y.; Kanekiyo, T. Blood-Brain Barrier Dysfunction and the Pathogenesis of Alzheimer's Disease. Int. J. Mol. Sci. 2017, 18, 1965. [CrossRef]

140. Santoro, J.D. Diminished Blood Pressure Profiles in Children with Down Syndrome. Hypertension 2020, 75, 819-825. [CrossRef]

141. Lao, P.J.; Gutierrez, J.; Keator, D.; Rizvi, B.; Banerjee, A.; Igwe, K.C.; Laing, K.K.; Sathishkumar, M.; Moni, F.; Andrews, H.; et al. Alzheimer-Related Cerebrovascular Disease in Down Syndrome. Ann. Neurol. 2020, 88, 1165-1177. [CrossRef]

142. Bayen, E.; Possin, K.L.; Chen, Y.; de Langavant, L.C.; Yaffe, K. Prevalence of Aging, Dementia, and Multimorbidity in Older Adults with Down Syndrome. JAMA Neurol. 2018, 75, 1399-1406. [CrossRef] [PubMed]

143. Walker, K.A.; Sharrett, A.R.; Wu, A.; Schneider, A.L.C.; Albert, M.; Lutsey, P.L.; Bandeen-Roche, K.; Coresh, J.; Gross, A.L.; Windham, B.G.; et al. Association of Midlife to Late-Life Blood Pressure Patterns with Incident Dementia. JAMA 2019, 322, 535-545. [CrossRef]

144. Höcht, C. Blood Pressure Variability: Prognostic Value and Therapeutic Implications. ISRN Hypertens. 2013, $2013,398485$. [CrossRef]

145. Parati, G.; Ochoa, J.E.; Lombardi, C.; Bilo, G. Blood Pressure Variability: Assessment, Predictive Value, and Potential as a Therapeutic Target. Curr. Hypertens. Rep. 2015, 17, 537. [CrossRef] [PubMed]

146. Ma, Y.; Wolters, F.J.; Chibnik, L.B.; Licher, S.; Ikram, M.K.; Hofman, A. Variation in blood pressure and long-term risk of dementia: A population-based cohort study. PLoS Med. 2019, 16, e1002933. [CrossRef] [PubMed] 
147. Matsumoto, A.; Satoh, M.; Kikuya, M.; Ohkubo, T.; Hirano, M.; Inoue, R.; Hashimoto, T.; Hara, A.; Hirose, T.; Obara, T.; et al. Day-to-day variability in home blood pressure is associated with cognitive decline: The Ohasama study. Hypertension 2014, 63, 1333-1338. [CrossRef]

148. Yano, Y.; Griswold, M.; Wang, W.; Greenland, P.; Lloyd-Jones, D.M.; Heiss, G.; Gottesman, R.F.; Mosley, T.H. Long-Term Blood Pressure Level and Variability From Midlife to Later Life and Subsequent Cognitive Change: The ARIC Neurocognitive Study. J. Am. Heart Assoc. 2018, 7, e009578. [CrossRef]

149. Godai, K.; SONIC Study Group; Kabayama, M.; Gondo, Y.; Yasumoto, S.; Sekiguchi, T.; Noma, T.; Tanaka, K.; Kiyoshige, E.; Akagi, Y. Day-to-day blood pressure variability is associated with lower cognitive performance among the Japanese community-dwelling oldest-old population: The SONIC study. Hypertens. Res. 2019, 43, 404-411. [CrossRef]

150. de Heus, R.A.A.; Rikkert, M.G.M.O.; Tully, P.J.; Lawlor, B.A.; Claassen, J.A.H.R. Blood Pressure Variability and Progression of Clinical Alzheimer Disease. Hypertension 2019, 74, 1172-1180. [CrossRef]

151. Oishi, E.; Ohara, T.; Sakata, S.; Fukuhara, M.; Hata, J.; Yoshida, D.; Shibata, M.; Ohtsubo, T.; Kitazono, T.; Kiyohara, Y.; et al. Day-to-Day Blood Pressure Variability and Risk of Dementia in a General Japanese Elderly Population: The Hisayama Study. Circulation 2017, 136, 516-525. [CrossRef]

152. Cho, N.; Hoshide, S.; Nishizawa, M.; Fujiwara, T.; Kario, K. Relationship Between Blood Pressure Variability and Cognitive Function in Elderly Patients with Good Blood Pressure Control. Am. J. Hypertens. 2017, 31, 293-298. [CrossRef]

153. Fujiwara, T.; Hoshide, S.; Kanegae, H.; Eguchi, K.; Kario, K. Exaggerated blood pressure variability is associated with memory impairment in very elderly patients. J. Clin. Hypertens. 2018, 20, 637-644. [CrossRef]

154. Liu, Z.; Zhao, Y.; Zhang, H.; Chai, Q.; Cui, Y.; Diao, Y.; Xiu, J.; Sun, X.; Jiang, G. Excessive variability in systolic blood pressure that is self-measured at home exacerbates the progression of brain white matter lesions and cognitive impairment in the oldest old. Hypertens. Res. 2016, 39, 245-253. [CrossRef]

155. McDonald, C.; Pearce, M.S.; Kerr, S.R.J.; Newton, J.L. Blood pressure variability and cognitive decline in older people: A 5-year longitudinal study. J. Hypertens. 2017, 35, 140-147. [CrossRef]

156. Nagai, M.; Hoshide, S.; Nishikawa, M.; Masahisa, S.; Kario, K. Visit-to-visit blood pressure variability in the elderly: Associations with cognitive impairment and carotid artery remodeling. Atherosclerosis 2014, 233, 19-26. [CrossRef]

157. Yıldırım, E.; Ermis, E.; Allahverdiyev, S.; Ucar, H.; Yavuzer, S.; Yavuzer, H.; Cengiz, M. Relationship between blood pressure variability and cognitive function in geriatric hypertensive patients with well-controlled blood pressure. Aging Clin. Exp. Res. 2019, 32, 93-98. [CrossRef] [PubMed]

158. Tsang, S.; Sperling, S.; Park, M.H.; Helenius, I.M.; Williams, I.C.; Manning, C. Blood Pressure Variability and Cognitive Function Among Older African Americans: Introducing a New Blood Pressure Variability Measure. Cogn. Behav. Neurol. 2017, 30, 90-97. [CrossRef] [PubMed]

159. van Middelaar, T.; van Dalen, J.W.; van Gool, W.A.; van den Born, B.H.; van Vught, L.A.; van Charante, E.P.M.; Richard, E. Visit-To-Visit Blood Pressure Variability and the Risk of Dementia in Older People. J. Alzheimers Dis. 2018, 62, 727-735. [CrossRef]

160. Qin, B.; Viera, A.J.; Muntner, P.; Plassman, B.L.; Edwards, L.; Adair, L.S.; Popkin, B.M.; Mendez, M.A. Visit-to-Visit Variability in Blood Pressure Is Related to Late-Life Cognitive Decline. Hypertension 2016, 68, 106-113. [CrossRef] [PubMed]

161. Alpérovitch, A.; Blachier, M.; Soumaré, A.; Ritchie, K.; Dartigues, J.-F.; Richard-Harston, S.; Tzourio, C. Blood pressure variability and risk of dementia in an elderly cohort, the Three-City Study. Alzheimer's Dement. 2013, 10, S330-S337. [CrossRef] [PubMed]

162. Epstein, N.U.; Lane, K.A.; Farlow, M.R.; Risacher, S.L.; Saykin, A.; Gao, S.; Initiative, T.A.D.N. Cognitive Dysfunction and Greater Visit-to-Visit Systolic Blood Pressure Variability. J. Am. Geriatr. Soc. 2013, 61, 2168-2173. [CrossRef] [PubMed]

163. Zhou, T.L.; Kroon, A.A.; Van Sloten, T.T.; Van Boxtel, M.P.; Verhey, F.R.; Schram, M.; Köhler, S.; Stehouwer, C.D.; Henry, R.M. Greater Blood Pressure Variability Is Associated with Lower Cognitive Performance. Hypertension 2019, 73, 803-811. [CrossRef]

164. Rouch, L.; Cestac, P. Visit-to-Visit Blood Pressure Variability Is Associated with Cognitive Decline and Incident Dementia: The S.AGES Cohort. Hypertension 2020, 76, 1280-1288. [CrossRef]

165. Sabayan, B.; Wijsman, L.W.; Foster-Dingley, J.C.; Stott, D.J.; Ford, I.; Buckley, B.M.; Sattar, N.; Jukema, J.W.; Van Osch, M.J.P.; Van Der Grond, J.; et al. Association of visit-to-visit variability in blood pressure with cognitive function in old age: Prospective cohort study. BMJ 2013, 347, f4600. [CrossRef]

166. Yoo, J.E.; Shin, D.W.; Han, K.; Kim, D.; Lee, S.-P.; Jeong, S.-M.; Lee, J.; Kim, S. Blood Pressure Variability and the Risk of Dementia. Hypertension 2020, 75, 982-990. [CrossRef]

167. Lattanzi, S.; Luzzi, S.; Provinciali, L.; Silvestrini, M. Blood pressure variability predicts cognitive decline in Alzheimer's disease patients. Neurobiol. Aging 2014, 35, 2282-2287. [CrossRef]

168. Sible, I.J.; Nation, D.A. Long-term blood pressure variability across the clinical and biomarker spectrum of Alzheimer's disease. J. Alzheimers Dis. 2020, 77, 1655-1669. [CrossRef] [PubMed]

169. Lattanzi, S.; Luzzi, S.; Provinciali, L.; Silvestrini, M. Blood Pressure Variability in Alzheimer's Disease and Frontotemporal Dementia: The Effect on the Rate of Cognitive Decline. J. Alzheimer's Dis. 2015, 45, 387-394. [CrossRef]

170. Lattanzi, S.; Brigo, F.; Vernieri, F.; Silvestrini, M. Visit-to-visit variability in blood pressure and Alzheimer's disease. J. Clin. Hypertens. 2018, 20, 918-924. [CrossRef]

171. Iadecola, C.; Parikh, N.S. Blood Pressure Ups and Downs Foreshadow Cerebral Microangiopathy. J. Am. Coll. Cardiol. 2020, 75, 2400-2402. [CrossRef] 
172. Zhou, T.L.; Rensma, S.P.; van der Heide, F.C.; Henry, R.M.; Kroon, A.A.; Houben, A.J.; Jansen, J.F.; Backes, W.H.; Berendschot, T.T.; Schouten, J.S.; et al. Blood pressure variability and microvascular dysfunction: The Maastricht Study. J. Hypertens. 2020, 38, 1541-1550. [CrossRef] [PubMed]

173. Anselmino, M.; Scarsoglio, S.; Saglietto, A.; Gaita, F.; Ridolfi, L. Transient cerebral hypoperfusion and hypertensive events during atrial fibrillation: A plausible mechanism for cognitive impairment. Sci. Rep. 2016, 6, 28635. [CrossRef] [PubMed]

174. De Leeuw, F.-E.; De Groot, J.C.; Oudkerk, M.; Kors, J.A.; Hofman, A.; Van Gijn, J.; Breteler, M.M. Atrial fibrillation and the risk of cerebral white matter lesions. Neurology 2000, 54, 1795-1801. [CrossRef] [PubMed]

175. Stefansdottir, H.; Arnar, D.O.; Aspelund, T.; Sigurdsson, S.; Jónsdóttir, M.K.; Hjaltason, H.; Launer, L.J.; Gudnason, V. Atrial Fibrillation is Associated with Reduced Brain Volume and Cognitive Function Independent of Cerebral Infarcts. Stroke 2013, 44, 1020-1025. [CrossRef] [PubMed]

176. Miao, C.-Y.; Su, D.-F. The importance of blood pressure variability in rat aortic and left ventricular hypertrophy produced by sinoaortic denervation. J. Hypertens. 2002, 20, 1865-1872. [CrossRef]

177. Nagai, M.; Dote, K.; Kato, M.; Sasaki, S.; Oda, N.; Kagawa, E.; Nakano, Y.; Yamane, A.; Higashihara, T.; Miyauchi, S.; et al Visit-to-Visit Blood Pressure Variability and Alzheimer's Disease: Links and Risks. J. Alzheimer's Dis. 2017, 59, 515-526. [CrossRef] [PubMed]

178. Yano, Y.; Reis, J.P.; Levine, D.A.; Bryan, R.N.; Viera, A.J.; Shimbo, D.; Tedla, Y.G.; Allen, N.B.; Schreiner, P.J.; Bancks, M.P.; et al. Visit-to-Visit Blood Pressure Variability in Young Adulthood and Hippocampal Volume and Integrity at Middle Age: The CARDIA Study (Coronary Artery Risk Development in Young Adults). Hypertension 2017, 70, 1091-1098. [CrossRef]

179. Ma, Y.; Tully, P.J.; Hofman, A.; Tzourio, C. Blood Pressure Variability and Dementia: A State-of-the-Art Review. Am. J. Hypertens. 2020, 33, 1059-1066. [CrossRef]

180. de Heus, R.A.A.; Reumers, S.F.I.; van der Have, A.; Tumelaire, M.; Tully, P.J.; Claassena, J.A.H.R. Day-to-Day Home Blood Pressure Variability is Associated with Cerebral Small Vessel Disease Burden in a Memory Clinic Population. J. Alzheimers Dis. 2020, 74, 463-472. [CrossRef] [PubMed]

181. Yamaguchi, Y.; Wada, M.; Sato, H.; Nagasawa, H.; Koyama, S.; Takahashi, Y.; Kawanami, T.; Kato, T. Impact of Ambulatory Blood Pressure Variability on Cerebral Small Vessel Disease Progression and Cognitive Decline in Community-Based Elderly Japanese. Am. J. Hypertens. 2014, 27, 1257-1267. [CrossRef] [PubMed]

182. Tully, P.J.; Debette, S.; Tzourio, C. The association between systolic blood pressure variability with depression, cognitive decline and white matter hyperintensities: The 3C Dijon MRI study. Psychol. Med. 2017, 48, 1444-1453. [CrossRef] [PubMed]

183. Tully, P.J.; Yano, Y.; Launer, L.J.; Kario, K.; Nagai, M.; Mooijaart, S.P.; Claassen, J.A.H.R.; Lattanzi, S.; Vincent, A.D.; Tzourio, C.; et al. Association between Blood Pressure Variability and Cerebral Small-Vessel Disease: A Systematic Review and Meta-Analysis. J. Am. Heart Assoc. 2020, 9, e013841. [CrossRef]

184. Ma, Y.; Yilmaz, P.; Bos, D.; Blacker, D.; Viswanathan, A.; Ikram, M.A.; Hofman, A.; Vernooij, M.W. Blood Pressure Variation and Subclinical Brain Disease. J. Am. Coll. Cardiol. 2020, 75, 2387-2399. [CrossRef]

185. Ma, Y. Blood Pressure Variability and Cerebral Small Vessel Disease: A Systematic Review and Meta-Analysis of Population-Based Cohorts. Stroke 2020, 51, 82-89. [CrossRef]

186. Gong, L.; Gu, Y.; Yu, Q.; Wang, H.; Zhu, X.; Dong, Q.; Xu, R.; Zhao, Y.; Liu, X. Prognostic Factors for Cognitive Recovery Beyond Early Poststroke Cognitive Impairment (PSCI): A Prospective Cohort Study of Spontaneous Intracerebral Hemorrhage. Front. Neurol. 2020, 11, 278. [CrossRef] [PubMed]

187. He, M.; Wang, J.; Liu, N.; Xiao, X.; Geng, S.; Meng, P.; Ji, N.; Sun, Y.; Xu, B.; Xu, Y.; et al. Effects of Blood Pressure in the Early Phase of Ischemic Stroke and Stroke Subtype on Poststroke Cognitive Impairment. Stroke 2018, 49, 1610-1617. [CrossRef]

188. Levine, D.A.; Galecki, A.T.; Okullo, D.; Briceño, E.M.; Kabeto, M.U.; Morgenstern, L.B.; Langa, K.M.; Giordani, B.; Brook, R.; Sanchez, B.N.; et al. Association of Blood Pressure and Cognition after Stroke. J. Stroke Cerebrovasc. Dis. 2020, $29,104754$. [CrossRef]

189. Yamamoto, Y.; Nagakane, Y.; Tomii, Y.; Akiguchi, I. High Morning and Bedtime Home Blood Pressures Strongly Predict for Post-Stroke Cognitive Impairment. J. Stroke Cerebrovasc. Dis. 2016, 25, 1856-1863. [CrossRef]

190. Sachdev, P.; Brodaty, H.; Valenzuela, M.; Lorentz, L.; Looi, J.; Berman, K.; Ross, A.; Wen, W.; Zagami, A. Clinical Determinants of Dementia and Mild Cognitive Impairment following Ischaemic Stroke: The Sydney Stroke Study. Dement. Geriatr. Cogn. Disord. 2006, 21, 275-283. [CrossRef]

191. Ihle-Hansen, H.; Thommessen, B.; Fagerland, M.; Oksengard, A.; Wyller, T.; Engedal, K.; Fure, B. Blood pressure control to prevent decline in cognition after stroke. Vasc. Health Risk Manag. 2015, 11, 311-316. [CrossRef] [PubMed]

192. Lu, Z.-H.; Li, J.; Li, X.-L.; Ding, M.; Mao, C.-J.; Zhu, X.-Y.; Liu, C.-F. Hypertension with Hyperhomocysteinemia Increases the Risk of Early Cognitive Impairment after First-Ever Ischemic Stroke. Eur. Neurol. 2020, 82, 75-85. [CrossRef] [PubMed]

193. Geng, S.; Liu, N.; Meng, P.; Ji, N.; Sun, Y.; Xu, Y.; Zhang, G.; He, X.; Cai, Z.; Wang, B.; et al. Midterm Blood Pressure Variability Is Associated with Poststroke Cognitive Impairment: A Prospective Cohort Study. Front. Neurol. 2017, 8, 365. [CrossRef]

194. Lee, J.-H.; Oh, E.; Oh, M.S.; Kim, C.; Jung, S.; Park, J.-H.; Kang, Y.; Yu, K.-H.; Lee, B.-C. Highly Variable Blood Pressure as a Predictor of Poor Cognitive Outcome in Patients with Acute Lacunar Infarction. Cogn. Behav. Neurol. 2014, 27, 189-198. [CrossRef] [PubMed]

195. Kim, Y. Blood pressure variability is related to faster cognitive decline in ischemic stroke patients: PICASSO subanalysis. Sci. Rep. 2021, 11, 5049. [CrossRef] 
196. Ishitsuka, K.; Kamouchi, M.; Hata, J.; Fukuda, K.; Matsuo, R.; Kuroda, J.; Ago, T.; Kuwashiro, T.; Sugimori, H.; Nakane, H.; et al. High Blood Pressure After Acute Ischemic Stroke Is Associated with Poor Clinical Outcomes. Hypertension 2014, 63, 54-60. [CrossRef] [PubMed]

197. Stead, L.G.; Gilmore, R.M.; Decker, W.W.; Weaver, A.L.; Brown, R.D. Initial emergency department blood pressure as predictor of survival after acute ischemic stroke. Neurology 2005, 65, 1179-1183. [CrossRef] [PubMed]

198. Bath, P.M.; Appleton, J.P.; Krishnan, K.; Sprigg, N. Blood Pressure in Acute Stroke. Stroke 2018, 49, 1784-1790. [CrossRef]

199. Powers, W.J.; Rabinstein, A.A.; Ackerson, T.; Adeoye, O.M.; Bambakidis, N.C.; Becker, K.; Biller, J.; Brown, M.; Demaerschalk, B.M.; Hoh, B.; et al. Guidelines for the Early Management of Patients with Acute Ischemic Stroke: 2019 Update to the 2018 Guidelines for the Early Management of Acute Ischemic Stroke: A Guideline for Healthcare Professionals From the American Heart Association/American Stroke Association. Stroke 2019, 50, e344-e418. [PubMed]

200. Sandset, E.C.; Anderson, C.S.; Bath, P.M.; Christensen, H.; Fischer, U.; Gąsecki, D.; Lal, A.; Manning, L.S.; Sacco, S.; Steiner, T.; et al. European Stroke Organisation (ESO) guidelines on blood pressure management in acute ischaemic stroke and intracerebral haemorrhage. Eur. Stroke J. 2021, 6, 23969873211012133. [CrossRef]

201. Kleindorfer, D.O.; Towfighi, A.; Chaturvedi, S.; Cockroft, K.M.; Gutierrez, J.; Lombardi-Hill, D.; Kamel, H.; Kernan, W.N.; Kittner, S.J.; Leira, E.C.; et al. 2021 Guideline for the Prevention of Stroke in Patients with Stroke and Transient Ischemic Attack: A Guideline From the American Heart Association/American Stroke Association. Stroke 2021. [CrossRef]

202. Ahmed, H.A.; Ishrat, T.; Pillai, B.; Fouda, A.; Sayed, M.A.; Eldahshan, W.; Waller, J.L.; Ergul, A.; Fagan, S.C. RAS modulation prevents progressive cognitive impairment after experimental stroke: A randomized, blinded preclinical trial. J. Neuroinflammation 2018, 15, 229. [CrossRef]

203. Jackson, L.; Dong, G.; Althomali, W.; Sayed, M.A.; Eldahshan, W.; Baban, B.; Johnson, M.H.; Filosa, J.; Fagan, S.C.; Ergul, A. Delayed Administration of Angiotensin II Type 2 Receptor (AT2R) Agonist Compound 21 Prevents the Development of Post-stroke Cognitive Impairment in Diabetes Through the Modulation of Microglia Polarization. Transl. Stroke Res. 2020, 11, 762-775. [CrossRef] [PubMed]

204. Oh, M.S.; Yu, K.-H.; Hong, K.-S.; Kang, D.-W.; Park, J.-M.; Bae, H.-J.; Koo, J.; Lee, J.; Lee, B.-C. Modest Blood Pressure Reduction with Valsartan in Acute Ischemic Stroke: A Prospective, Randomized, Open-Label, Blinded-End-Point Trial. Int. J. Stroke 2015, 10, 745-751. [CrossRef]

205. Sandset, E.C.; Bath, P.; Boysen, G.; Jatuzis, D.; Kõrv, J.; Lüders, S.; Murray, G.D.; Richter, P.S.; Roine, R.; Terént, A.; et al. The angiotensin-receptor blocker candesartan for treatment of acute stroke (SCAST): A randomised, placebo-controlled, double-blind trial. Lancet 2011, 377, 741-750. [CrossRef]

206. Li, N.-C.; Le, K.; Whitmer, R.; Kivipelto, M.; Lawler, E.; Kazis, L.; Wolozin, B. Use of angiotensin receptor blockers and risk of dementia in a predominantly male population: Prospective cohort analysis. BMJ 2010, 340, b5465. [CrossRef]

207. Anderson, C.; Teo, K.; Gao, P.; Arima, H.; Dans, A.; Unger, T.; Commerford, P.; Dyal, L.; Schumacher, H.; Pogue, J.; et al. Renin-angiotensin system blockade and cognitive function in patients at high risk of cardiovascular disease: Analysis of data from the ONTARGET and TRANSCEND studies. Lancet Neurol. 2011, 10, 43-53. [CrossRef]

208. Group, T.P.C. Effects of Blood Pressure Lowering with Perindopril and Indapamide Therapy on Dementia and Cognitive Decline in Patients with Cerebrovascular Disease. Arch. Intern. Med. 2003, 163, 1069-1075.

209. Ölmestig, J.; Marlet, I.R.; Hansen, R.H.; Rehman, S.; Krawcyk, R.S.; Rostrup, E.; Lambertsen, K.L.; Kruuse, C. Tadalafil may improve cerebral perfusion in small-vessel occlusion stroke-a pilot study. Brain Commun. 2020, 2, 11. [CrossRef] [PubMed]

210. ENOS Trial Investigators. Efficacy of nitric oxide, with or without continuing antihypertensive treatment, for management of high blood pressure in acute stroke (ENOS): A partial-factorial randomised controlled trial. Lancet 2015, 385, 617-628. [CrossRef]

211. O’Brien, E.C.; Greiner, M.A.; Xian, Y.; Fonarow, G.; Olson, D.M.; Schwamm, L.H.; Bhatt, D.L.; Smith, E.E.; Maisch, L.; Hannah, D.; et al. Clinical Effectiveness of Statin Therapy After Ischemic Stroke: Primary Results From the Statin Therapeutic Area of the Patient-Centered Research Into Outcomes Stroke Patients Prefer and Effectiveness Research (PROSPER) Study. Circulation 2015, 132, 1404-1413. [CrossRef]

212. Parfenov, V.; Belavina, G.R.; Vakhnina, N.V.; Gusev, V.V.; Levin, I.; Markin, S.P.; Starchina, I. Use of omaron in patients with post-stroke cognitive disorders. Zhurnal Nevrol. Psikhiatrii Korsakova 2009, 109, 41-45.

213. Shozawa, H.; Oguchi, T.; Tsuji, M.; Yano, S.; Kiuchi, Y.; Ono, K. Supratherapeutic concentrations of cilostazol inhibits $\beta$-amyloid oligomerization in vitro. Neurosci. Lett. 2018, 677, 19-25. [CrossRef] [PubMed]

214. Lee, Y.S.; Park, S.Y.; Heo, H.J.; Lee, W.S.; Hong, K.W.; Kim, C.D. Multitarget-directed cotreatment with cilostazol and aripiprazole for augmented neuroprotection against oxidative stress-induced toxicity in HT22 mouse hippocampal cells. Eur. J. Pharmacol. 2019, 857, 172454. [CrossRef]

215. Senda, J.; Ito, K.; Kotake, T.; Kanamori, M.; Kishimoto, H.; Kadono, I.; Nakagawa-Senda, H.; Wakai, K.; Katsuno, M.; Nishida, Y.; et al. Cilostazol use is associated with FIM cognitive improvement during convalescent rehabilitation in patients with ischemic stroke: A retrospective study. Nagoya J. Med. Sci. 2019, 81, 359-373. [PubMed]

216. Lim, J.-S.; Kwon, S.U.; Yu, K.-H.; Yu, S.; Park, J.-H.; Lee, B.-C.; Oh, M.S.; Kim, Y.-J.; Rha, J.-H.; Hwang, Y.-H.; et al. Cilostazol and Probucol for Cognitive Decline after Stroke: A Cognitive Outcome Substudy of the PICASSO Trial. J. Stroke 2021, 23, 128-131. [CrossRef] [PubMed] 
217. Chang, W.H.; Park, Y.H.; Ohn, S.H.; Park, C.-H.; Lee, P.K.W.; Kim, Y.-H. Neural correlates of donepezil-induced cognitive improvement in patients with right hemisphere stroke: A pilot study. Neuropsychol. Rehabil. 2011, 21, 502-514. [CrossRef] [PubMed]

218. Narasimhalu, K.; Effendy, S.; Sim, C.H.; Lee, J.M.; Chen, I.; Hia, S.B.; Xue, H.L.; Corrales, M.P.; Chang, H.M.; Wong, M.C.; et al. A randomized controlled trial of rivastigmine in patients with cognitive impairment no dementia because of cerebrovascular disease. Acta Neurol. Scand. 2010, 121, 217-224. [CrossRef]

219. Gagnon, D.J.; Leclerc, A.M.; Riker, R.R.; Brown, C.S.; May, T.; Nocella, K.; Cote, J.; Eldridge, A.; Seder, D.B. Amantadine and Modafinil as Neurostimulants During Post-stroke Care: A Systematic Review. Neurocritical Care 2020, 33, 283-297. [CrossRef]

220. Du, Y. Effect of acupuncture treatment on post-stroke cognitive impairment: A randomized controlled trial. Medicine 2020, 99, e23803. [CrossRef]

221. Zhou, L.; Wang, Y.; Qiao, J.; Wang, Q.M.; Luo, X. Acupuncture for Improving Cognitive Impairment After Stroke: A Meta-Analysis of Randomized Controlled Trials. Front. Psychol. 2020, 11, 549265. [CrossRef]

222. Shih, C.-C.; Yeh, C.-C.; Hu, C.-J.; Lane, H.-L.; Tsai, C.-C.; Chen, T.-L.; Liao, C.-C. Risk of dementia in patients with nonhaemorrhagic stroke receiving acupuncture treatment: A nationwide matched cohort study from Taiwan's National Health Insurance Research Database. BMJ Open 2017, 7, e013638. [CrossRef] [PubMed]

223. Cumming, T.B.; Tyedin, K.; Churilov, L.; Morris, M.E.; Bernhardt, J. The effect of physical activity on cognitive function after stroke: A systematic review. Int. Psychogeriatrics 2011, 24, 557-567. [CrossRef] [PubMed]

224. Teuschl, Y.; Matz, K.; Brainin, M. Prevention of post-stroke cognitive decline: A review focusing on lifestyle interventions. Eur. J. Neurol. 2012, 20, 35-49. [CrossRef]

225. Reale, M.; Costantini, E.; Jagarlapoodi, S.; Khan, H.; Belwal, T.; Cichelli, A. Relationship of Wine Consumption with Alzheimer's Disease. Nutrients 2020, 12, 206. [CrossRef] [PubMed]

226. Moroney, J.T.; Bagiella, E.; Tatemichi, T.K.; Paik, M.C.; Stern, Y.; Desmond, D.W. Dementia after stroke increases the risk of long-term stroke recurrence. Neurology 1997, 48, 1317-1325. [CrossRef]

227. Fisicaro, F.; Lanza, G.; Pennisi, M.; Vagli, C.; Cantone, M.; Pennisi, G.; Ferri, R.; Bella, R. Moderate Mocha Coffee Consumption Is Associated with Higher Cognitive and Mood Status in a Non-Demented Elderly Population with Subcortical Ischemic Vascular Disease. Nutrients 2021, 13, 536. [CrossRef] [PubMed]

228. Wang, X.; Li, J.; Qian, L.; Zang, X.-F.; Zhang, S.-Y.; Jin, J.-L.; Zhu, X.-L.; Zhang, X.-B.; Wang, Z.-Y.; Xu, Y. Icariin promotes histone acetylation and attenuates post-stroke cognitive impairment in the central cholinergic circuits of mice. Neuroscience 2013, 236, 281-288. [CrossRef]

229. Pennisi, M.; Lanza, G.; Cantone, M.; D’Amico, E.; Fisicaro, F.; Puglisi, V.; Vinciguerra, L.; Bella, R.; Vicari, E.; Malaguarnera, G Acetyl-L-Carnitine in Dementia and Other Cognitive Disorders: A Critical Update. Nutrients 2020, 12, 1389. [CrossRef] [PubMed]

230. Yang, Y.; Choi, H.; Lee, C.-N.; Kim, Y.B.; Kwak, Y.T. A Multicenter, Randomized, Double-blind, Placebo-controlled Clinical Trial for Efficacy of Acetyl-L-carnitine in Patients with Dementia Associated with Cerebrovascular Disease. Dement. Neurocognitive Disord. 2018, 17, 1-10. [CrossRef]

231. Li, Y.; Luo, H.; Yu, Q.; Yin, L.; Li, K.; Li, Y.; Fu, J. Cerebral Functional Manipulation of Repetitive Transcranial Magnetic Stimulation in Cognitive Impairment Patients After Stroke: An fMRI Study. Front. Neurol. 2020, 11, 977. [CrossRef]

232. Yin, M.; Liu, Y.; Zhang, L.; Zheng, H.; Peng, L.; Ai, Y.; Luo, J.; Hu, X. Effects of rTMS Treatment on Cognitive Impairment and Resting-State Brain Activity in Stroke Patients: A Randomized Clinical Trial. Front. Neural Circuits 2020, 14, 563777. [CrossRef]

233. Hara, T.; Shanmugalingam, A.; McIntyre, A.; Burhan, A. The Effect of Non-Invasive Brain Stimulation (NIBS) on Attention and Memory Function in Stroke Rehabilitation Patients: A Systematic Review and Meta-Analysis. Diagnostics 2021, 11, 227. [CrossRef] [PubMed]

234. Ma, J.; Zhang, Z.; Su, Y.; Kang, L.; Geng, D.; Wang, Y.; Luan, F.; Wang, M.; Cui, H. Magnetic stimulation modulates structural synaptic plasticity and regulates BDNF-TrkB signal pathway in cultured hippocampal neurons. Neurochem. Int. 2013, 62, 84-91. [CrossRef]

235. Sun, L.; Lu, H.; Zhang, T.; Wen, M. Impact of Repetitive Transcranial Magnetic Stimulation on Post-Stroke Dysmnesia and the Role of BDNF Val66Met SNP. Med. Sci. Monit. 2015, 21, 761-768. [CrossRef] [PubMed]

236. Hong, J.; Chen, J.; Li, C.; An, D.; Tang, Z.; Wen, H. High-Frequency rTMS Improves Cognitive Function by Regulating Synaptic Plasticity in Cerebral Ischemic Rats. Neurochem. Res. 2021, 46, 276-286. [CrossRef] [PubMed]

237. Shen, X.; Liu, M.; Cheng, Y.; Jia, C.; Pan, X.; Gou, Q.; Liu, X.; Cao, H.; Zhang, L. Repetitive transcranial magnetic stimulation for the treatment of post-stroke depression: A systematic review and meta-analysis of randomized controlled clinical trials. J. Affect. Disord. 2017, 211, 65-74. [CrossRef]

238. Baylan, S.; Swann-Price, R.; Peryer, G.; Quinn, T. The effects of music listening interventions on cognition and mood post-stroke: A systematic review. Expert Rev. Neurother. 2016, 16, 1241-1249. [CrossRef] [PubMed]

239. Särkämö, T.; Tervaniemi, M.; Laitinen, S.; Forsblom, A.; Soinila, S.; Mikkonen, M.; Autti, T.; Silvennoinen, H.M.; Erkkilä, J.; Laine, M.; et al. Music listening enhances cognitive recovery and mood after middle cerebral artery stroke. Brain 2008, 131, 866-876. [CrossRef] [PubMed] 